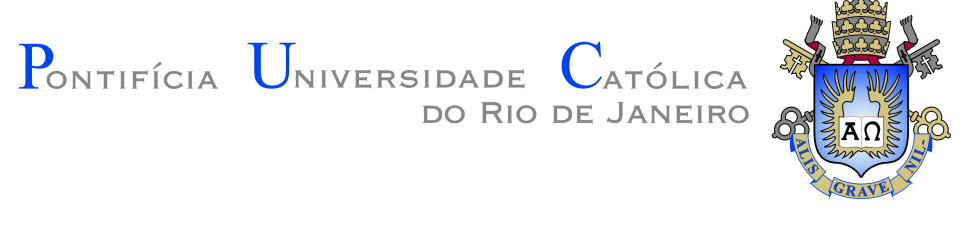

Danielle de Macedo

\title{
Estratégias para garantir viabilidade e consistência temporal no planejamento da produção de processos de manufatura discreta
}

Dissertação de Mestrado

Dissertação apresentada como requisito parcial para obtenção do grau de Mestre pelo Programa de Pós-graduação em Engenharia de Produção do Departamento de Engenharia Industrial da PUCRio.

Orientador : Prof. Bruno Fanzeres dos Santos Coorientadora: Prof. Paula Medina Maçaira Louro 

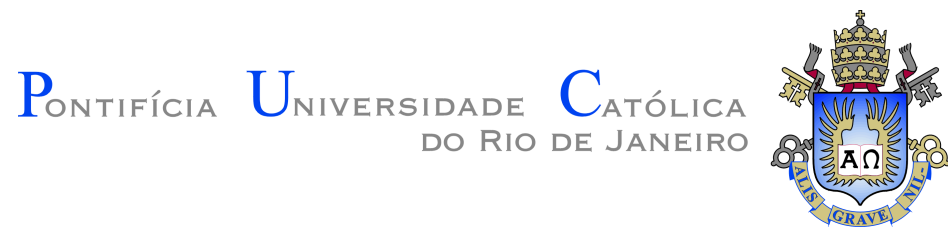

Danielle de Macedo

Estratégias para garantir viabilidade e consistência temporal no planejamento da produção de processos de manufatura discreta

Dissertação apresentada como requisito parcial para obtenção do grau de Mestre pelo Programa de Pós-Graduação em Engenharia de Produção da PUC-Rio. Aprovada pela Comissão Examinadora abaixo:

Prof. Bruno Fanzeres dos Santos

Orientador

Departamento de Engenharia Industrial - PUC-Rio

Prof. Paula Medina Maçaira Louro

Coorientadora

Departamento de Engenharia Industrial - PUC-Rio

Prof. Luciana de Souza Pessoa

Departamento de Engenharia Industrial - PUC-Rio

Prof. Rafael Martinelli Pinto

Departamento de Engenharia Industrial - PUC-Rio

Prof. Fernando Marques de Almeida Nogueira UFJF 
Todos os direitos reservados. É proibida a reprodução total ou parcial do trabalho sem autorização da universidade, do autor e do orientador.

\section{Danielle de Macedo}

Graduada em Engenharia de Produção pela Universidade Federal de Juiz de Fora.

Ficha Catalográfica

Macedo, Danielle de

Estratégias para garantir viabilidade e consistência temporal no planejamento da produção de processos de manufatura discreta / Danielle de Macedo; orientador: Bruno Fanzeres dos Santos; coorientadora: Paula Medina Maçaira Louro. 2021.

v., 53 f. : il. color. ; $30 \mathrm{~cm}$

Dissertação (mestrado) - Pontifícia Universidade Católica do Rio de Janeiro, Departamento de Engenharia Industrial, 2021.

Inclui bibliografia

1. Engenharia Industrial - Teses. 2. Planejamento da Produção. 3. Pedido de Material. 4. Sequenciamento da Produção. 5. Decisão sob Incerteza. 6. Consistência Temporal. I. Santos, Bruno Fanzeres dos. II. Louro, Paula Medina Maçaira. III. Pontifícia Universidade Católica do Rio de Janeiro. Departamento de Engenharia Industrial. IV. Título. 


\section{Agradecimentos}

Aos meus pais Nemias Soares de Macedo e Vera Lucia de Assis Macedo, que sempre me incentivaram e me deram todo o suporte para que eu continuasse meus estudos.

A todos os docentes do Programa de pós-graduação da Engenharia Industrial da PUC-RIO, em especial ao meu orientador Bruno Fânzeres dos Santos, que além do suporte técnico me incentivou muito e demonstrou extrema paciência com minhas inquietações acadêmicas durante toda a trajetória, e a minha coorientadora Paula Medina Maçaira Louro, que me ajudou a melhorar o trabalho.

A Universidade Federal de Juiz de Fora (UFJF), em particular ao professor Fernando Marques de Almeida Nogueira, que fez toda a ponte para a concretização do MINTER, programa pelo qual ingressei no curso.

A Deus, que tem me sustentado até aqui e permitiu que tudo isso fosse possível. O presente trabalho foi realizado com apoio da Coordenação de Aperfeiçoamento de Pessoal de Nível Superior - Brasil (CAPES) - Código de Financiamento 001 


\section{Resumo}

Macedo, Danielle de; Santos, Bruno Fanzeres dos; Louro, Paula Medina Maçaira. Estratégias para garantir viabilidade e consistência temporal no planejamento da produção de processos de manufatura discreta. Rio de Janeiro, 2021. 53p. Dissertação de Mestrado - Departamento de Engenharia Industrial, Pontifícia Universidade Católica do Rio de Janeiro.

Tradicionalmente, em indústrias de produção de peças discretas, no nível tático do planejamento da produção, é calculado o plano mestre de produção (Master Production Scheduling - MPS), que estabelece a quantidade de cada bem a ser produzida por período. Com o MPS em mãos, a necessidade de matéria-prima é levantada e o requerimento de material é realizado levandose em consideração o lead time de chegada das peças, que está relacionado com o modal de transporte previamente definido pela empresa. Mais próximo da operação, o sequenciamento dos jobs é feito com o objetivo de atender ao planejamento do MPS. Normalmente, esses quatro problemas - definição do modal de transporte, elaboração do plano mestre de produção, definição do momento de compra de materiais e sequenciamento da produção - são tratados em momentos diferentes e, muitas vezes, de forma determinística. Neste trabalho é avaliado o impacto financeiro e operacional de realizar o planejamento de forma determinística e segregada. Em particular, avaliase: (i) o impacto da estocasticidade e co-otimização do planejamento da produção e das decisões de compra e (ii) a viabilidade do sequenciamento. Para (i) é proposto um modelo de otimização estocástica de dois estágios que co-otimiza as decisões de volume de produção, momentos de compra e modal de transporte. Para (ii) restrições de sequenciamento de jobs são adicionadas através de uma heurística e de um modelo de programação matemática. Avaliações empíricas são feitas por meio de dois experimentos numéricos com dados realistas de uma empresa do setor automobilístico. Para (i) observamos uma redução de custo de 7\% na operação para o ano de 2018 (ano do planejamento) e cerca de $3,5 \%$ para 5000 cenários simulados (out-ofsample), comparando o modelo de dois estágios proposto com o procedimento normalmente adotado na indústria. Para (ii) também observamos uma redução de $8 \%$ no custo de operação de 2018 , e de $9,6 \%$ para 50 cenários simulados (outof-sample), em relação ao modelo proposto em (i) e 13\% no custo de operação de 2018 e 15,6\% para 50 cenários simulados (out-of-sample), em comparação com o modelo típico da indústria.

\section{Palavras-chave}


Planejamento da Produção; Pedido de Material; Sequenciamento da Produção; Decisão sob Incerteza; Consistência Temporal. 


\section{Abstract}

Macedo, Danielle de; Santos, Bruno Fanzeres dos (Advisor); Louro, Paula Medina Maçaira (Co-Advisor). Strategies to Ensure Planning Feasibility and Time Consistency in Discrete Manufacturing Production Processes. Rio de Janeiro, 2021. 53p. Dissertação de Mestrado - Departamento de Engenharia Industrial, Pontifícia Universidade Católica do Rio de Janeiro.

Traditionally, in discrete manufacturing industries, at the tactical level of production planning, the master production scheduling (MPS) is calculated, which establishes the quantity of each good to be produced per period. With the MPS in hand, the need for raw material is raised and the material requirement is carried out taking into account the lead time arrival of the parts, which is related to the transport mode previously defined by the company. Closer to the operation, the jobs scheduling is done with the purpose of meeting MPS planning. Typically, these four problems - definition of the transportation mode, preparation of master production scheduling, definition of the time to purchase materials and job scheduling - are dealt with at different times and often in a deterministic way. In this work we evaluate the financial and operational impact of carrying out the planning in a deterministic and segregated way. In particular, we assess: (i) the impact of stochasticity and co-optimization of production planning and purchasing decisions and (ii) the feasibility of job scheduling. For (i) a two-stage stochastic optimization model is proposed that co-optimizes production volume decisions, purchase moments and transportation mode. For (ii) sequencing constraints of jobs are added through a heuristic and a mathematical programming model. Empirical assessments are made through two numerical experiments with realistic data from a discrete manufacturing company. For (i) we observed $7 \%$ cost reduction in the operation for the year 2018 (planning year) and approximately 3.5\% for 5000 simulated scenarios (out-of-sample), comparing the proposed two-stage model with the procedure typically adopted in the industry. For (ii) we also observed a reduction of $8 \%$ in the 2018 operation cost, and $9.6 \%$ for 50 simulated scenarios (out-of-sample), in relation to the model proposed in (i) and $13 \%$ in the 2018 operation cost and $15.6 \%$ for 50 simulated scenarios (out-of-sample), compared to the typical industry model.

\section{Keywords}

Production Planning; Material Ordering; Scheduling; Decision-Making under Uncertainty; Time Consistency. 


\section{Sumário}

1 Introdução 11

2 Planejamento da Produção 14

2.1 Planejamento tático da produção 14

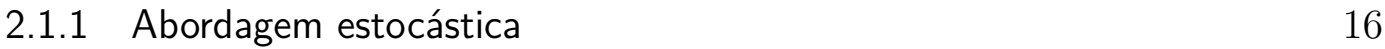

2.2 Planejamento operacional da produção 18

2.3 Requerimento de material 21

$\begin{array}{ll}2.4 & \text { Sistemas ERP }\end{array}$

3 Planejamento Integrado de Produção e Compra sob Incerteza na Demanda 24

3.1 Modelo de Planejamento Determinístico da Produção com Regra Posterior para Requerimento de Material 26

3.2 Modelo Estocástico em Dois Estágios para o Planejamento da Produção com Requerimento Integrado de Material 28

3.3 Desenho do experimento 29

$\begin{array}{ll}\text { 3.3.1 Dados de entrada } & 29\end{array}$

3.3.2 Previsão e Simulação dos Cenários de Demanda 30

3.3.3 Algoritmo de comparação 31

3.4 Resultados Numéricos 32

3.4.1 Análise Baseada na Demanda Real Observada para 2018

3.4.2 Análise Probabilística para $2018 \quad 34$

3.4.3 Análise de sensibilidade 34

4 Planejamento Integrado de Produção, Compra e Sequenciamento de jobs, sob Incerteza na Demanda 38

4.1 Modelo Estocástico em Dois Estágios para o Planejamento da Produção com Requerimento Integrado de Material e Viabilidade do Sequenciamento da Produção

4.2 Heurística para Verificação da Viabilidade do Sequenciamento da Produção Durante Etapa de Planejamento 42

4.3 Desenho do experimento 43

4.3.1 Dados de entrada 44

4.3.2 Previsão e Simulação dos Cenários de Demanda 44

4.3.3 Algoritmo de comparação 45

4.4 Resultados Numéricos $\quad 45$

4.4.1 Análise Baseada na Demanda Real Observada para 2018

4.4.2 Análise Probabilística para $2018 \quad 47$

5 Conclusão e Trabalhos Futuros $\quad 49$

$\begin{array}{ll}\text { Referências bibliográficas } & 50\end{array}$ 


\section{Lista de figuras}

Figura 3.1 Série histórica de venda de veículos do Ipeadata. 31

Figura 3.2 Custo total planejado e o efetivamente observado usando os dados reais para 2018 para cada um dos modelos analisados.

Figura 3.3 Parcelas do custo total comparando o planejado com o efetivamente observado usando os dados reais para 2018 entre cada modelo analisado.

Figura 3.4 Distribuição acumulada (amostral) da diferença percentual entre o custo total do modelo estocástico e determinístico

Figura 3.5 Diferença média por configuração dos parâmetros

Figura 4.1 Custo total planejado e o efetivamente observado usando os dados reais para 2018 para cada um dos modelos analisados.

Figura 4.2 Distribuição acumulada (amostral) da diferença percentual entre o custo total dos modelos com sequenciamento M. 03 e M.04 em relação aos modelos sem sequenciamento determinístico M.01 e estocástico M.02 


\section{Lista de tabelas}

Tabela 2.1 Lista com a nomenclatura contendo os conjuntos, parâmetros e variáveis utilizadas no problema MLCLSP.

Tabela 2.2 Diferentes formas para se considerar incerteza no planejamento da produção.

Tabela 2.3 Lista com a nomenclatura contendo os conjuntos, parâmetros e variáveis utilizadas no problema job-shop.

Tabela 2.4 Políticas para definição de lotes de compra e sua periodicidade.

Tabela 3.1 Lista com a nomenclatura contendo os conjuntos, parâmetros e variáveis utilizadas.

Tabela 4.1 Lista com a nomenclatura contendo os conjuntos, parâmetros e variáveis utilizados.

Tabela 4.2 Lista com a nomenclatura contendo os conjuntos, parâmetros e variáveis utilizadas no problema job-shop.

Tabela 4.3 Tempo de execução e memória utilizada para rodar uma instância de cada modelo. 


\section{Introdução}

O planejamento da produção está relacionado com o gerenciamento dos recursos produtivos necessários para transformar matéria-prima em produtos finais, de forma a satisfazer os clientes da maneira mais eficiente possível (Pochet, 2001). De acordo com Madroñero (2014), o planejamento da produção é a resposta para "onde", "quando" e "quanto" produzir de forma a encontrar a máxima eficiência do sistema. Em função do horizonte de tempo considerado, diversos autores, como Salomon (1991) e Min (2002), classificam o planejamento da produção em estratégico (5 a 10 anos), tático (alguns meses a 2 anos) e operacional (1 ou 2 semanas). No presente trabalho o foco está no planejamento tático/operacional, considerando a existência de um desenho de sistema e capacidades pré-definidos.

Tradicionalmente, em indústrias de produção de peças discretas, no nível tático do planejamento de produção é feito o cálculo do plano mestre de produção (Master Production Scheduling - MPS), que tem por objetivo determinar a quantidade de cada bem a ser produzida por período. O MPS pode ser elaborado tanto com heurísticas como através de um modelo de programação matemática. De maneira geral, o plano mestre de produção é elaborado tendo como base um problema de dimensionamento de lotes. Existem várias revisões sobre esse tipo de problema como em (Buschkuhl, 2010) onde é apresentada uma revisão dos métodos de solução, e em (Jans, 2008) onde é apresentada a modelagem do problemas de dimensionamento de lotes para várias extensões industriais.

$\mathrm{Na}$ indústria, esse problema é majoritariamente tratado de forma determinística, tomando-se previsões para incertezas. Na academia, existem alguns trabalhos que consideram a incerteza no problema. Hu (2016) fornece um modelo estocástico de dois estágios para a definição de lotes e momentos de produção considerando incerteza na demanda e na qualidade dos materiais, $\mathrm{Hu}$ (2018) resolve um problema semelhante ao anterior, mas multiestágio. Ambos os estudos consideram produtos com apenas um nível de produção.

Com o MPS em mãos, a necessidade de matéria-prima é levantada. Para o requerimento de material exitem algumas políticas que podem ser seguidas. Uma opção é a cada período comprar a demanda do momento (lote-a-lote), 
outra possibilidade é admitir um estoque inicial e comprar a demanda menos o estoque (JIT), essas são chamadas estratégias não parametrizadas.

$\mathrm{Na}$ busca por contornar as múltiplas incertezas inerentes ao processo, é comum se utilizar de estratégias chamadas parametrizadas, nas quais se define um ponto de ressuprimento e um estoque máximo. Essa política poderia ser usada até mesmo para definir o volume de produção, mas a dificuldade está no fato dela admitir que a demanda, durante o período de lead time de produção/pedido, segue uma distribuição de demanda estacionária e conhecida, o que não é verdadeiro no caso que estamos estudando (veja os trabalhos (Chen, 1994), (Chen, 2000), (Castellano, 2018), (Yue, 2013) e (Wei, 2020) para mais detalhes).

Seja qual for a estratégia utilizada na gestão do estoque, para se definir o momento do requerimento da matéria-prima deve-se levar em conta o lead time de chegada das peças, que está relacionado com o modal de transporte previamente definido pela empresa. Tipicamente indústrias que precisam de uma maior flexibilidade optam por modais mais ágeis como o aéreo ou rodoviário e empresas que conseguem lidar com uma menor flexibilidade preferem um modal de transporte de mais baixo custo, como o marítimo ou ferroviário.

Mais perto da operação, o sequenciamento dos jobs é feito. Este tem por propósito atender o planejamento de produção do período, conforme determinado pelo MPS.

O sequenciamento dos jobs também é uma etapa do planejamento tipicamente resolvida deterministicamente. Chaudhry (2016) faz uma revisão no tema. Entre os artigos que consideram incerteza, destaca-se o trabalho de Sha (2021), onde é desenvolvido um modelo estocástico multiestágio de sequenciamento de jobs co-otimizado com o requerimento de materiais, sob incerteza no tempo de processamento e consumo de recursos.

Tradicionalmente esses quatro problemas - definição de modal de transporte, elaboração do plano mestre de produção, definição do momento de compra dos materiais e sequenciamento da produção - são tratados em momentos distintos e muitas vezes de forma determinística. No presente trabalho será avaliado o impacto financeiro e operativo de realizar o planejamento de forma determinística e segregada. Em particular, iremos avaliar: (i) Impacto da estocasticidade e co-otimização de planejamento de produção e decisões de compra e (ii) Viabilidade do sequenciamento na operação. Para (i) é proposto um modelo de otimização estocástica dois estágios que co-otimiza as decisões de volumes de produção, momentos de compra e modal de transporte e para (ii) restrições de sequenciamento de jobs são adicionadas através de uma heu- 
rística e de um modelo de programação matemática. É importante ressaltar que nesse trabalho a escolha do modal de transporte é no âmbito operacional. Os possíveis modais de transporte e suas características serão tratados como parâmetros do problema.

Os modelos MLCLSP (Multi-Level Capacitated Lot-Sizing Problem) para planejamento de produção, conforme apresentado em (Billington, 1983) e o modelo para job-shop apresentado em (Lageweg, 1977), serão utilizados como base para as metodologias propostas nessa dissertação.

As avaliações serão feitas através de dois experimentos numéricos com dados realistas de uma empresa do setor automotivo. Para (i) observa-se 7\% de redução de custo na operação para o ano de 2018 (ano em que o planejamento foi realizado) e 3,57\% para 5000 cenários simulados (out-ofsample), comparando o modelo proposto com o tipicamente realizado na indústria. Para (ii) observa-se ainda uma redução de $8 \%$ no custo da operação de 2018, e 9,6\% para 50 cenários simulados (out-of-sample), em relação ao modelo proposto em (i) e 13\% no custo da operação de 2018 e 15,6\% para 50 cenários simulados (out-of-sample), em relação modelo típico da indústria.

O trabalho está organizado da seguinte forma: No capítulo 2 é apresentado o referencial teórico da dissertação. No capítulo 3 é proposto um modelo de otimização sob incerteza em dois estágios para co-otimização das ordens de compra e elaboração do MPS que é comparado com a abordagem (determinística e em duas etapas) tipicamente utilizada na indústria. No capítulo 4 restrições de sequenciamento são adicionadas ao modelo proposto de duas formas e os modelos são novamente comparados entre si. A conclusão é feita no capítulo 5. 


\section{2}

\section{Planejamento da Produção}

Planejamento da produção está relacionado com o gerenciamento dos recursos produtivos necessários para transformar matéria-prima em produtos finais de forma a satisfazer os clientes da maneira mais eficiente possível (Pochet, 2001). De acordo com Madroñero (2014), o planejamento da produção é a resposta para "onde", "quando" e "quanto" produzir de forma a encontrar a máxima eficiência do sistema.

O planejamento de produção pode ser decomposto de acordo com o horizonte de tempo considerado. Diversos autores, como Salomon (1991) e Min (2002), classificam o planejamento de produção em estratégico, tático e operacional.

De forma geral, o planejamento estratégico está preocupado com um horizonte de 5 a 10 anos, o tático com um horizonte de alguns meses a 2 anos e o planejamento operacional com a operação das próximas uma ou duas semanas.

Enquanto o planejamento estratégico afeta a configuração do sistema, buscando respostas a perguntas do tipo onde construir uma fábrica e qual a capacidade produtiva instalar em maquinário, o planejamento tático se preocupa no melhor uso dos recursos já disponíveis, respondendo questões como quantidades a serem produzidas, níveis de inventário e fluxo de materiais. Por fim, o planejamento operacional, tendo como entrada a solução do planejamento tático, busca detalhar o melhor sequenciamento das atividades, tamanho de lotes e rotas de veículos.

$\mathrm{Na}$ presente dissertação busca-se responder o que, quanto e quando produzir e comprar, considerando um desenho de sistema definido, por isso apenas os planejamentos tático e operacional serão analisados.

\section{1}

\section{Planejamento tático da produção}

O planejamento tático da produção visa a elaboração de um plano mestre de produção (MPS) que é um documento que define, por período, quais produtos serão produzidos e em quais quantidades. A princípio este não é 
um plano rígido, é um plano dinâmico que pode ser alterado quando houver alterações na demanda ou capacidade.

O MPS pode ser elaborado tanto com heurísticas quanto através de um modelo matemático. Várias revisões, como (Karimia, 2003), (Quadt, 2007), (Jans, 2008) e (Buschkuhl, 2010) apresentam métodos de solução e extensões industriais nesse tema.

Esse trabalho considera o problema MLCLSP (the multi-level capacitated lot-sizing problem) como base para se calcular o plano mestre de produção, conforme apresentado em (Billington, 1983). Nas restrições (2-1)-(2-7) o modelo é detalhado. Seus conjuntos, parâmetros e variáveis estão descritos na tabela 2.1 .

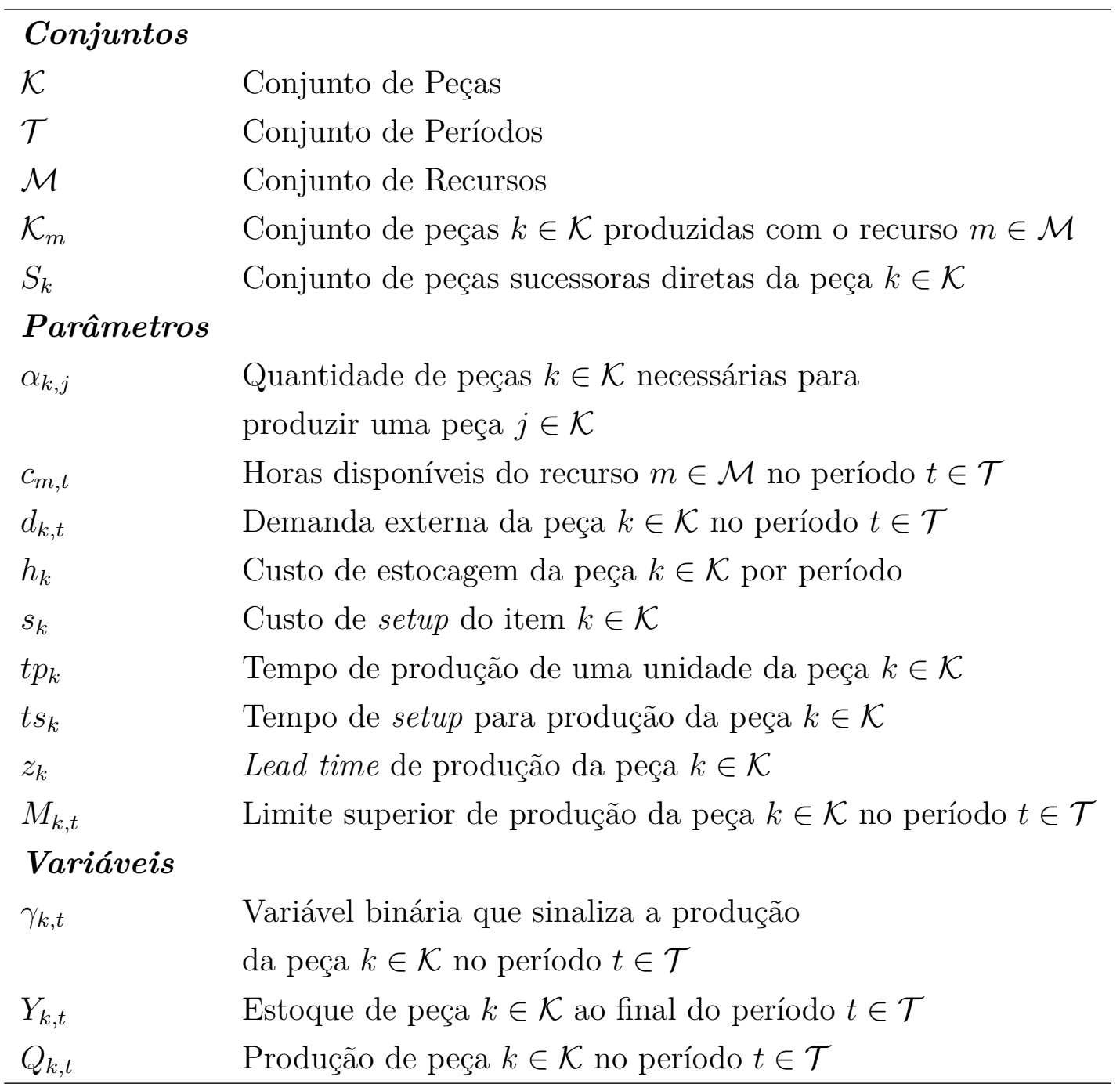

Tabela 2.1: Lista com a nomenclatura contendo os conjuntos, parâmetros e variáveis utilizadas no problema MLCLSP. 


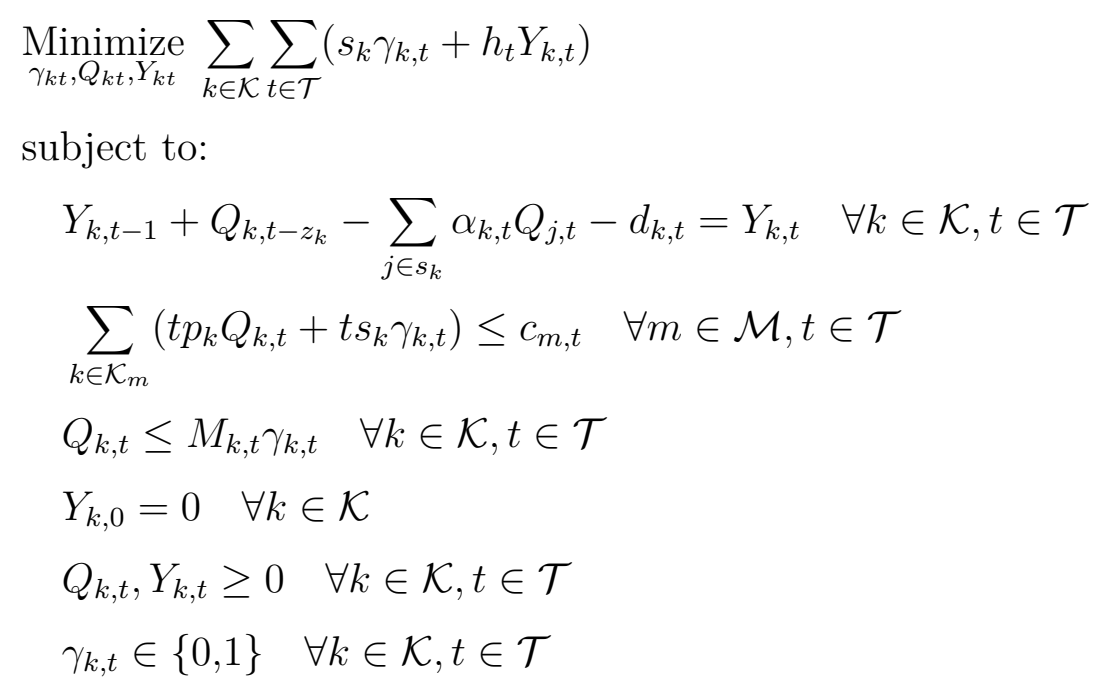

A função objetivo (2-1) minimiza a soma do custo total com setup e estoque para todos os itens em todos os períodos, o modelo considera que o custo de produção é o mesmo em todos os períodos, motivo pelo qual o custo de produção não precisa entrar na função objetivo do problema. A restrição (2-2) estabelece que a demanda de cada período deve ser atendida sem permissão de atraso. Para cada item $k \in \mathcal{K}$ é considerado um lead time de produção $z_{k}$. A restrição (2-3) garante que a capacidade de cada recurso não será excedida por período, considerando que cada peça é fabricada utilizando-se de exatamente um recurso e que toda vez que houver fabricação de uma peça $k \in \mathcal{K}$ haverá gasto com setup, mesmo se já houvesse produção da peça no período anterior. A restrição (2-4) serve para garantir que o custo de setup será contabilizado caso haja produção da peça $k \in \mathcal{K}$ no período $t \in \mathcal{T}$. Na restrição $(2-5)$ os estoques iniciais são definidos em zero, na restrição (2-6) temos a não-negatividade das variáveis de produção e estoque e na restrição (2-7) a definição de $\gamma_{k, t}$ como variável binária.

Em (Suerie, 2003) é apresentado uma forma de considerar transições e, assim, se contabilizar apenas uma vez o custo de setup quando um item é produzido de forma contínua entre um período e outro. Em Shapiro (1993) é apresentada uma formulação do modelo considerando-se o uso de hora-extra.

\subsection{1}

\section{Abordagem estocástica}

Existem na literatura diferentes formas para se considerar incerteza no planejamento da produção. Mula (2006) os classifica em 5 grupos (Modelos conceituais, modelos de cobertura, modelos baseados em inteligência artificial, modelos analíticos e modelos de simulação) e 26 subgrupos, que são apresen- 
tados na tabela 2.2.

\section{Modelos Conceituais}

Fatores de produção

Estoques de Segurança

lead timess de segurança

Cobertura

Overplanning

Planejamento de requisitos de linha

Flexibilidade

\section{Modelos baseados em IA}

Sistemas espertos

Aprendizagem por reforço

Teoria de conjunto Fuzzy

Lógica Fuzzy

Redes neurais

Algorítimos genéticos

Sistemas multi-agentes

\section{Modelos Analíticos}

Processos hierárquicos

Programação matemática

(LP, MILP, NLD,

$\mathrm{DP}$ and MOP)

Programação estocástica

Aproximações determinísticas

Transformadas de Laplace

Processos de decisão Markovianos

\section{Modelos de Simulação}

Técnicas de Monte Carlo

Distribuições de probabilidade

Métodos heurísticos

Parâmetros congelados

Teoria da fila

Sistemas dinâmicos

Tabela 2.2: Diferentes formas para se considerar incerteza no planejamento da produção.

Uma forma de lidar com incerteza no planejamento da produção é aplicando uma ordem de decisão através de uma regra de controle de inventário estocástico (Axsater, 1994).

$$
p=P(x)= \begin{cases}Y F(C S-x), & x<C S \\ 0, & x \geq C S .\end{cases}
$$

Onde, p é a quantidade de produto que deve ser encomendada/fabricada, x o status do nível de inventário, CS é o ponto crítico do estoque (calculado através da distribuição de probabilidade da demanda durante o lead time de entrega/fabricação do produto) e YF um fator de correção de permissão de falha na produção.

Inderfurth (2007) mostra que o estoque crítico CS é um $\alpha$-quantil da distribuição de demanda, de acordo com a solução do problema clássico do jornaleiro, e $\mathrm{p}(\mathrm{x})$ é facilmente calculado caso as incertezas apresentem uma distribuição uniforme ou exponencial, ou não haja incerteza na fabricação do produto. Para o caso em que há incerteza na produção e em que as distribuições 
não são uniformes ou exponenciais, são analisadas algumas possibilidades apresentadas na literatura e escolhida a que se comportou melhor. Embora esse trabalho forneça uma boa solução em termos de tratamento do risco, considera-se apenas um planejamento da produção em que cada período deve produzir exatamente a quantidade necessária no período (lot-for-lot), não possibilitando produção adiantada ou atrasos, tal desenho pode ser interessante para empresas com uma demanda regular, mas muito custoso para empresas com alta sazonalidade, devido a necessidade de altos gastos com hora-extra ou contratação e demissão. (Chen, 1994), (Chen, 2000), (Castellano, 2018), (Yue, 2013) e (Wei, 2020) são outros trabalhos que apresentam soluções na mesma linha.

Dentre os trabalho encontrados, os que estão mais na linha com o proposto nessa dissertação são Hu (2016), que fornece um modelo estocástico de dois estágios para a definição de lotes e momentos de produção, considerando incerteza na demanda e na qualidade dos materiais, e $\mathrm{Hu}$ (2018), que resolve um problema semelhante ao anterior, mas multiestágio. Ambos os estudos consideram produtos com apenas um nível de produção.

\section{2}

\section{Planejamento operacional da produção}

Conforme foi falado no início do capítulo, o planejamento operacional da produção tem como entrada a solução do planejamento tático e está relacionado a atividades como o sequenciamento das operações.

De acordo com Shapiro (1993) a complexidade do sequenciamento jobshop gera grandes e difíceis modelos de otimização combinatoriais, o que faz com que, na prática, os modelos sejam usados junto com métodos analíticos e heurísticos mais com o intuito de encontrar bons sequenciamentos do que se encontrar a solução ótima.

Inicialmente será apresentado um modelo base de sequenciamento jobshop e em seguida algumas possíveis extensões para o mesmo. Como ponto de partida, será considerado o modelo proposto em (Lageweg, 1977) que está descrito nas restrições $(2-8)-(2-14)$. 


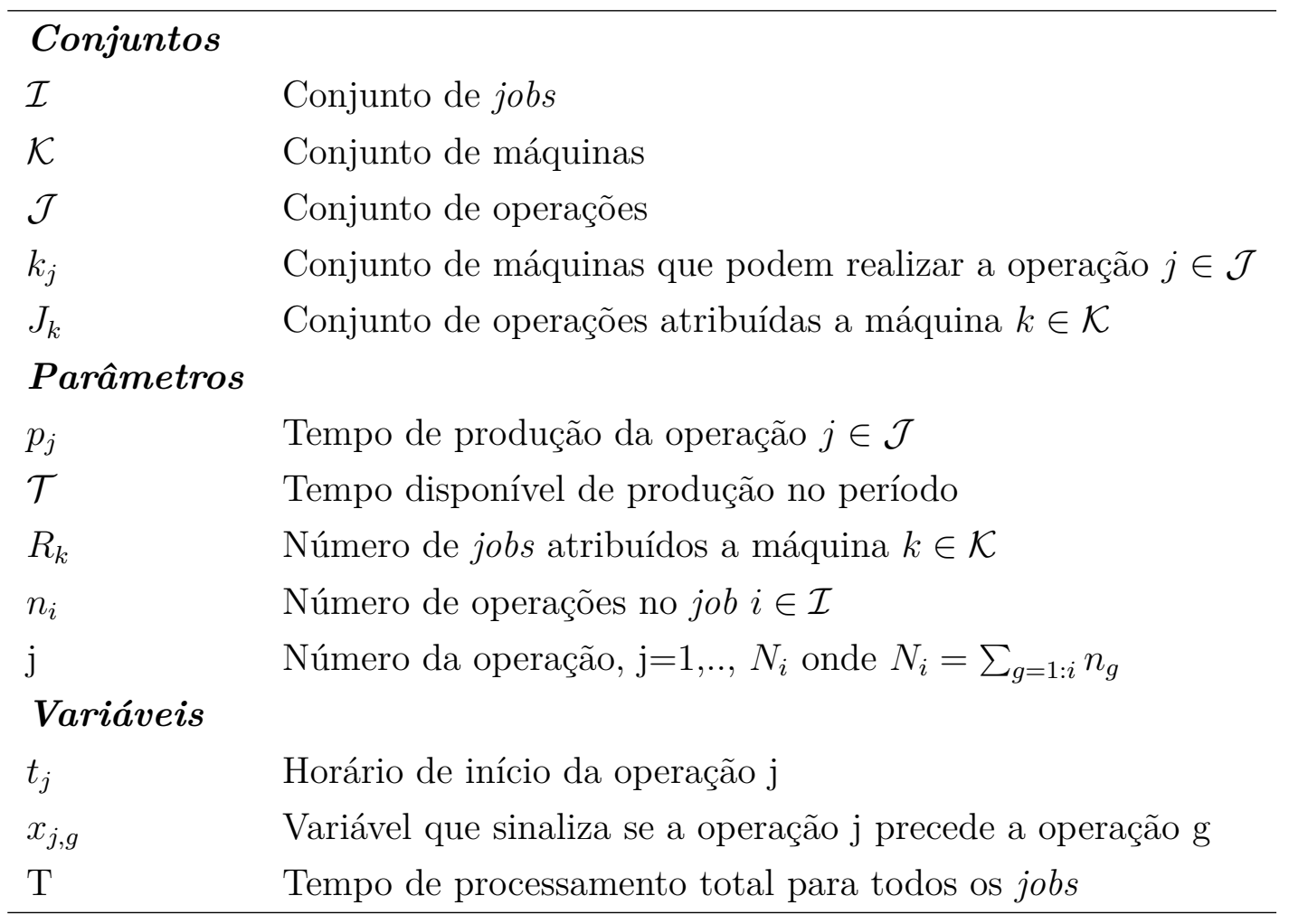

Tabela 2.3: Lista com a nomenclatura contendo os conjuntos, parâmetros e variáveis utilizadas no problema job-shop.

$$
\begin{aligned}
& \underset{t_{j}, x_{j, g}, T}{\operatorname{Minimize}} T \\
& \text { subject to: } \\
& \qquad t_{j} \geq t_{j-1}+p_{j-1} \quad \forall i \in I, \forall j \in\left\{N_{i-1}+2, \ldots, N_{i}\right\} \\
& \quad T \geq t_{N_{i}}+p_{N_{i}} \quad \forall i \in I \\
& t_{g} \geq t_{j}+p_{j} x_{j, g}-\mathcal{T}\left(1-x_{j, g}\right) \quad \forall g, j \in J_{k}, \quad \forall k \in \mathcal{K} \\
& t_{j} \geq t_{g}+p_{g}\left(1-x_{j, g}\right)-\mathcal{T} x_{j, g} \quad \forall g, j \in J_{k}, \quad \forall k \in \mathcal{K} \\
& x_{j, g} \in\{0,1\} \\
& \quad T, t_{j} \geq 0 \quad \forall j \in \mathcal{J}
\end{aligned}
$$

A função objetivo (2-8) busca minimizar o tempo de finalização do último job. A restrição (2-9) assegura que os processos realizados em um job serão feitos ordenadamente (é assumido aqui que todos os jobs passam por uma ordem fixa de processamento que não pode ser alterada). A restrição (2-10) captura o tempo de finalização do último job. As restrições (2-11) e (2-12) impõem que dois processos não podem ser realizados simultaneamente em uma mesma máquina. Por fim, a restrição (2-13) define $x_{j, g}$ como variável 
binária e a restrição (2-14) impõe a não-negatividade das variáveis de tempo. (?) apresenta algumas extensões que podem ser realizadas no modelo.

Caso um job $j \in I$ possua ordem de precedência para apenas algumas operações, a restrição (2-9) pode ser substituída pela restrição (2-15), onde $P g$ é o conjunto de operações $j$ sucessoras a operação $g$.

$$
t_{g}-t_{j} \geq p_{j} \quad \forall g \in \mathcal{J}, \forall j \in P g
$$

No modelo base foi considerado uma atribuição de tarefas para cada máquina feita a priori. Caso o processo seja mais flexível, com mais máquinas podendo realizar o mesmo processo, tal escolha pode ser considerada como variável no modelo, o que é possível substituindo as restrições (2-11) e (2-12) pelas restrições (2-16), (2-17), (2-18) e (2-19).

$$
\begin{aligned}
& t_{g} \geq t_{j}+p_{j} x_{j, g, k}-\mathcal{T}\left(1-x_{j, g, k}\right)-\mathcal{T}\left(2-y_{j, k}-y_{g, k}\right) \\
& t_{j} \geq t_{g}+p_{g}\left(1-x_{j, g, k}\right)-\mathcal{T} x_{j, g, k}-\mathcal{T}\left(2-y_{j, k}-y_{g, k}\right) \\
& \sum_{k \in K_{j}} y_{j, k}=1 \quad \forall j \\
& y_{j, k} \in\{0,1\} \quad \forall j \forall k
\end{aligned}
$$

O modelo base não considera tempo de setup, o que pode ser bastante significativo dependendo do par ( $\mathrm{j}, \mathrm{g})$ considerado em uma sequência de operações. Shapiro (1993) argumenta que uma variável binária seria necessária para capturar cada opção de troca $(\mathrm{j}, \mathrm{g})$, o que torna uma extensão de programação mista-inteira (MILP), do problema base, excessivamente grande. Para superar esse problema, é sugerido que o problema seja visto como uma coleção de problemas do caixeiro viajante, um para cada máquina.

No modelo base buscou-se através da função objetivo minimizar o tempo de finalização de todas as tarefas, entretanto, pode-se optar por diferentes objetivos como, por exemplo, minimizar o custo de produção considerando que cada job possui diferentes custos dependendo da máquina a qual este foi enviado.

Mesmo sem o uso de nenhuma das extensões citadas Shapiro (1993) diz que o modelo base, por si só, já é grande e complexo. Buscando contornar essa dificuldade computacional, diversos autores, como (Lageweg, 1977) e (Adams, 1988), propuseram técnicas de solução baseadas em combinações de esquemas branch-and-bound, heurísticas e limites inferiores baseados em relaxações fáceis de otimizar. 
Devido a sua complexidade, o sequenciamento de jobs é tipicamente resolvido de maneira determinística, mas existem alguns trabalhos na literatura que o fazem de forma estocástica. Existem estudos (por exemplo, (Matsuura, 1993), (Lawrence, 1997), (Liu, 1998), (Calleja, 2014), (Gao, 2015) e (Karunakaran, 2017)) que usam regras de despacho para agendamento sob incerteza e regras de despacho especificamente para lidar com a incerteza, como a "maior variância primeiro" (Pinedo, 1987) e "a menor variância primeiro" (Pinedo, 2007). Alguns estudos, mais na linha do que será proposto nessa dissertação, buscam definir o melhor sequenciamento, dado a incerteza, apenas considerando as restrições da operação, sem utilizar-se de regras pré-estabelecidas. Destaca-se o trabalho de Sha (2021), onde é desenvolvido um modelo estocástico multiestágio de sequenciamento de jobs co-otimizado com o requerimento de material, sob incerteza no tempo de processamento e consumo de recursos.

\section{3}

\section{Requerimento de material}

De acordo com o plano mestre de produção é feito o requerimento da compra da matéria-prima para produção. Existem diversas políticas descritas na literatura que podem ser utilizadas para se estabelecer tamanhos de lote de compra e sua periodicidade, a ideia é minimizar conjuntamente o custo de pedido e de estoque sem que haja falta de peças no momento estipulado para a produção.

Na tabela 2.4 alguns procedimentos descritos na literatura são apresentados. Veja (Nydick, 1989), (Jeunet, 2000), (Simpson, 2001) e (Kropp, 2007) para mais detalhes.

Além das políticas citadas na tabela 2.4, para se contornar as incertezas do processo, pode-se utilizar de estratégias parametrizadas, nas quais se define um ponto de ressuprimento e um estoque máximo, conforme descrito na seção 2.1.1. 


\section{Procedimentos}

Fixed Order Quantity (FOQ)

Pedir uma quantidade fixa no ponto de ressuprimento (quantidade pode ser calculada de via EOQ (Economic Order Quantity))

Lot-for-lot (L4L)

Pedir exatamente o que será usado em cada período

Single-lot

Juntar toda a necessidade de um período e pedir de uma vez

Period of Supply

Um período (ex.: x semanas) é especificado e a necessidade de

todo o período é reunida formando um único lote

Period Order Quantity (POQ)

Os pedidos são feitos considerando-se múltiplos do EOQ, para isso calcula-se a demanda média por período e os pedidos são feitos periodicamente de forma a atender a demanda de produção.

Least Unit Cost (LUC)

O tamanho do lote é determinado de forma que a demanda para os próximos "n" períodos seja atendida. "n" deve ser tal que se minimize o custo com pedido e estoque por unidade.

Least Total Cost (LTC)

Os pedidos são feitos de forma que os custos de pedido se aproximem aos custos de transporte.

Silver Meal (SM)

Metodologia para se resolver o LUC de forma iterativa. O processo

é iniciado com um "n" que vai aumentando até que o custo por período comece a aumentar.

UMSOQPVAD

Processo dinâmico com uma ideia similar ao SM, usado pelo SAP.

Tabela 2.4: Políticas para definição de lotes de compra e sua periodicidade.

\section{4}

\section{Sistemas ERP}

O ERP (Enterprise Resource Planning) é um sistema de informação que agrupa todos os dados e processos de uma organização.

Sistemas MRP (Material Requirement Planning) foram originalmente desenhados na década de 70, sendo os antecessores dos atuais sistemas ERP. Inicialmente, tendo como entrada o MPS e a estrutura dos produtos (BOM - Bill of Material) esses sistemas tinham apenas a função de desdobrar as 
necessidades de aquisição de matéria prima e de produção dos produtos intermediários.

Já na década de 80 foi acrescentado a esses sistemas informações de capacidade e o gerenciamento de recursos como mão-de-obra e maquinário, utilizando-se agora o nome de MRP II. Em seguida começaram-se a agregar ao MRP novos sistemas contemplando áreas como finanças, compras e vendas e recursos humanos. Schollaert (1994) introduz funções financeiras ao MRP, chamando-o de MRP III, a nomenclatura ERP ganhou força na década de 90.

De acordo com Ptak (2011), embora inicialmente a formulação dos sistemas MRP não estivessem associadas com nenhuma forma de otimização, a maximização dos lucros e restrições de suprimento, demanda e recursos disponíveis foram gradualmente incorporadas ao sistema, sendo o modelo MLCLSP um dos principais meios para os cálculos das necessidades do MRP.

Mula (2012) discorre que o processo mais habitual para o planejamento da produção é o cálculo do MPS determinando as quantidades de cada bem a ser produzida em um horizonte de planejamento e em seguida as ordens de compra associadas as peças e matérias-prima são levantadas. É argumentado que os sistemas de planejamento de produção não consideram simultaneamente aspectos relacionados ao trasporte de peças e matéria prima a suas instalações, o que pode levar a resultados inviáveis em termos de transporte, fazendo com que a solução encontrada pelo MRP tenha que ser alterada manualmente, não gerando uma otimização dos recursos. Tendo isso em vista, o artigo sugere um modelo conceitual, chamando-o de MRP IV, para o planejamento de produção integrado ao planejamento do transporte. O artigo apresenta apenas o conceito da ideia, sem entrar em detalhes sobre como operacionalizá-la, além disso o conceito de transporte a que este se refere está mais relacionado com a otimização dos custos com o número de viagens necessárias, rotas, não tratando da utilização do modal de transporte como uma maneira de lidar com as incertezas na demanda, em vista à maior flexibilidade para a indústria, conforme proposto na presente dissertação. 


\section{Planejamento Integrado de Produção e Compra sob Incerteza na Demanda}

Este capítulo busca apresentar uma ideia a respeito dos impactos no custo por se considerar a demanda de forma determinística e por se determinar o pedido de material de forma segregada ao planejamento da produção.

O capítulo está organizado da seguinte forma: Nas duas primeiras seções os modelos de planejamento em indústrias com produção discreta são apresentados, na seção 3.3 é apresentado o desenho do experimento e na seção 3.4 são descritos os resultados numéricos.

As metodologias aqui descritas são baseadas no modelo MLCLSP descrito na seção 2.1. Na Seção 3.1, é apresentado o modelo de planejamento da produção na sua forma mais tradicional, simulando a prática das indústrias. Mais especificamente, o modelo tem como entrada a previsão de demanda para o horizonte de planejamento considerado e como saída as quantidades a serem produzidas, os inventários e as faltas por período. Para se estabelecer as ordens de pedido, com suas quantidades e modos de transporte, uma heurística é descrita levando em consideração os resultados do modelo de planejamento. Será considerado que a empresa tem a sua disposição dois modais de transporte, um modal "MA" mais ágil e um modal "MB" de mais baixo custo. A política da empresa é sempre comprar por meio do modal "MB" e apenas utilizar o modal "MA" caso haja necessidade, devido ao curto período de tempo até o começo da produção. Posteriormente, na Seção 3.2, um modelo de planejamento da produção sob incerteza, proposto neste trabalho, é apresentado. Iremos lançar mão da abordagem tipicamente utilizada na literatura técnica em problemas de otimização sob incerteza e considerar um conjunto de possíveis cenários de demanda para o horizonte de planejamento, construindo um modelo de programação estocástica em dois estágios. Além disso, adicionamos ao modelos as variáveis de ordem de pedido que serão cootimizadas junto ao planejamento.

Para uma melhor compreensão dos modelos apresentados neste capítulo, na Tabela 3.1, é apresentada uma lista de nomenclatura contendo os índices, parâmetros e variáveis utilizados. Por simplicidade, neste trabalho, foi considerado um período unitário para o lead time de pedido pelo modal "MA", 
Capítulo 3. Planejamento Integrado de Produção e Compra sob Incerteza na Demanda

de dois períodos para o pedido pelo modal "MB" e nulo para o lead time de produção.

\begin{tabular}{ll}
\hline Conjuntos & \\
$\mathcal{I}$ & Conjunto de Peças \\
$\mathcal{T}$ & Conjunto de períodos \\
$\mathcal{K}$ & Conjunto de faixas de produção \\
$\mathcal{M}$ & Conjunto de máquinas/estações de trabalho \\
& \\
Omega & Conjunto de cenários de demanda \\
$\mathcal{J}_{m}$ & Conjunto de peças $i \in \mathcal{I}$ produzidas com o recurso $m \in \mathcal{M}$ \\
$S_{i}$ & Conjunto de peças sucessoras diretas da peça $i \in \mathcal{I}$
\end{tabular}

\section{Parâmetros}

$\alpha_{i, j} \quad$ Quantidade de peças $i \in \mathcal{I}$ necessárias para a produção

da peça $j \in \mathcal{I}$

\section{$\tau_{m, k}^{m}$}

$d_{i, t, \omega}$

Tempo disponível do recurso $m \in \mathcal{M}$ na faixa de produção $k \in \mathcal{K}$

Demanda externa da peça $i \in \mathcal{I}$, no período $t \in \mathcal{T}$,

no cenário $\omega \in \Omega$

Custo de estocagem da peça $i \in \mathcal{I}$ por período

$c_{i}^{s}$

Custo de setup de máquina para começar a produção

do produto $i \in \mathcal{I}$

Custo de produção da peça $i \in \mathcal{I}$ na faixa de produção $k \in \mathcal{K}$

Custo de déficit da peça $i \in \mathcal{I}$

Custo do pedido "MA" da peça $i \in \mathcal{I}$

Custo do pedido marítimo da peça $i \in \mathcal{I}$

Tempo de produção de uma unidade da peça $i \in \mathcal{I}$

Tempo de setup para produção da peça $i \in \mathcal{I}$

$l_{i}^{p}$

Lead time de produção da peça $i \in \mathcal{I}$

Lead time do pedido "MA" de peça $i \in \mathcal{I}$

$l_{i}^{b}$

Lead time do pedido marítimo de peça $i \in \mathcal{I}$

$\bar{Q}_{i}$

Produção máxima da peça $i \in \mathcal{I}$ por período

$\bar{X}_{i}^{a}$

Pedido máximo da peça $i \in \mathcal{I}$ por modo "MA"

$\bar{X}_{i}^{a}$

Pedido máximo da peça $i \in \mathcal{I}$ por modo "MB"

$P_{\omega}$

Probabilidade de ocorrência do cenário $\omega \in \Omega$

$Y_{i}^{0}$

Inventário inicial da peça $i \in \mathcal{I}$ 
Capítulo 3. Planejamento Integrado de Produção e Compra sob Incerteza na Demanda

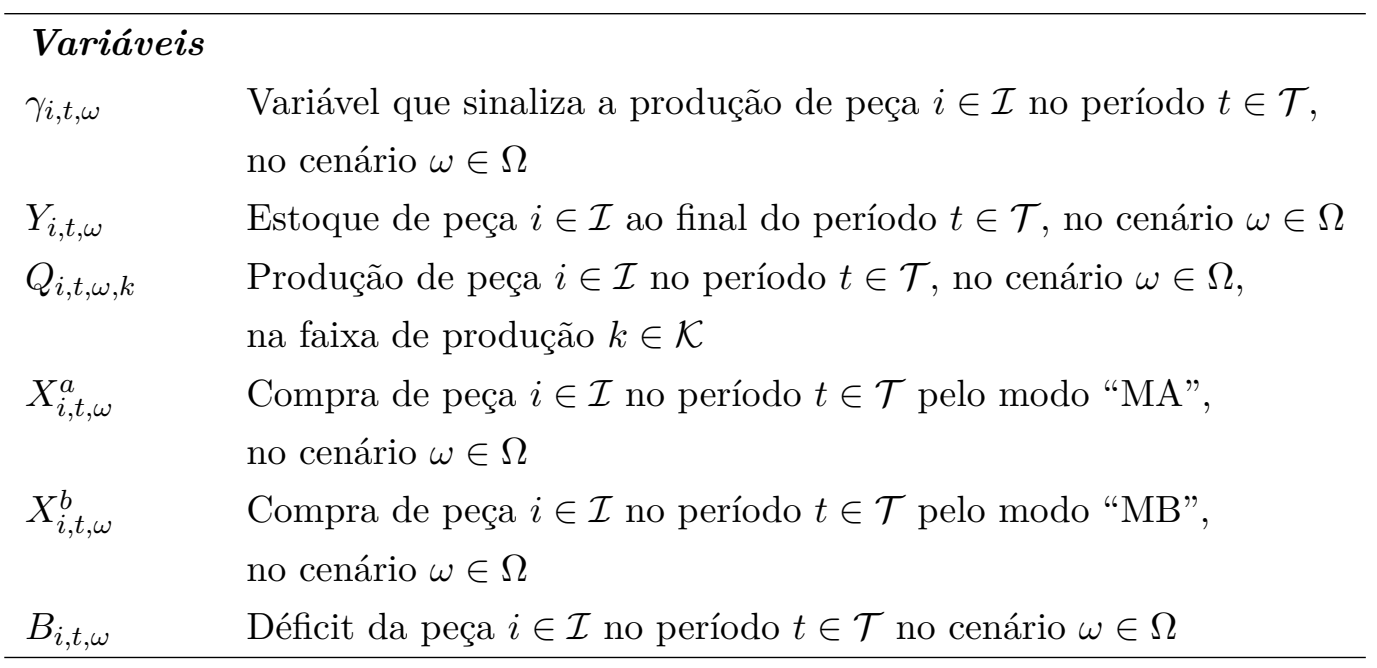

Tabela 3.1: Lista com a nomenclatura contendo os conjuntos, parâmetros e variáveis utilizadas.

\section{1}

Modelo de Planejamento Determinístico da Produção com Regra Posterior para Requerimento de Material

Na sua essência o modelo de planejamento da produção busca minimizar a soma dos custos da operação (produção, setup, estoque, falta, etc.) sujeito às restrição de capacidade e balanço de peças. O modelo é apresentado nas equações (3-1)-(3-9) a seguir.

$$
\underset{\gamma_{i t}, Q_{i t k}, Y_{i t}, B_{i t}}{\operatorname{Minimize}} \sum_{t \in \mathcal{T}} \sum_{i \in \mathcal{I}}\left(c_{i}^{s} \gamma_{i, t}+c_{i}^{e} Y_{i, t}+\sum_{k \in K} c_{i, k}^{p} Q_{i, t, k}+c_{i}^{d} B_{i, t}\right)
$$

subject to:

$$
\begin{array}{cr}
Y_{i}^{0}+\sum_{k \in K} Q_{i, t, k}-\sum_{j \in S_{i}} \alpha_{i, j}\left(\sum_{k \in K} Q_{j, t, k}\right)-Y_{i, t}+B_{i, t}=d_{i, t} \\
\forall i \in \mathcal{I}, t \in \mathcal{T} \mid t=1 ; \\
Y_{i, t-1}+\sum_{k \in K} Q_{i, t, k}-\sum_{j \in S_{i}} \alpha_{i, j}\left(\sum_{k \in K} Q_{j, t, k}\right)-Y_{i, t}+B_{i, t}=d_{i, t}, \\
\forall i \in \mathcal{I}, t \in \mathcal{T} \mid t \neq 1 ; \\
Y_{i}^{0}+Q_{i, t, 1}-\sum_{j \in S_{i}} \alpha_{i, j} Q_{j, t, 1} \geq 0, \\
\forall i \in \mathcal{I}, t \in \mathcal{T} \mid t=1 ; \\
Y_{i, t-1}+Q_{i, t, 1}-\sum_{j \in S_{i}} \alpha_{i, j} Q_{j, t, 1} \geq 0, \\
\sum_{j \in \mathcal{J}_{m}}\left(\tau_{j}^{p} Q_{j, t, k}+\tau_{j, k}^{s} \gamma_{j, t}\right) \leq \tau_{m, k}^{m}, \\
\sum_{k \in K} Q_{i, t, k} \leq \bar{Q}_{i} \gamma_{i, t}, \\
\forall i \in \mathcal{I}, t \in \mathcal{T} \mid t \neq 1 ;
\end{array}
$$




$$
\begin{aligned}
& Q_{i, t, k}, Y_{i, t}, B_{i, t} \geq 0, \\
& \gamma_{i, t} \in\{0,1\},
\end{aligned}
$$

$\forall i \in \mathcal{I}, t \in \mathcal{T}$

$\forall i \in \mathcal{I}, t \in \mathcal{T}$.

O modelo (3-1)-(3-9) é um modelo (determinístico) de programação linear inteiro-misto que tem como objetivo minimizar (equação (3-1)) a soma do custo total com setup, estoque, produção e falta para todos os itens em todos os períodos. As restrições (3-2) e (3-3) garantem o balanceamento da produção, sem possibilidade de atraso. Caso a demanda de algum item em certo período $t \in \mathcal{T}$ não seja atendida, é contabilizada a falta do produto. As restrições (3-4) e (3-5) foram necessárias apenas pelo fato de estarmos considerando mais de uma faixa de produção $k \in \mathcal{K}$ e são necessárias para assegurar que não haja inversão da ordem da produção de peças com relação de precedência uma pra outra. A restrição (3-6) garante que a capacidade de cada recurso (que pode ser entendido como uma máquina ou estação de trabalho) não será excedida por período. Considera-se que cada peça seja fabricada utilizando-se de exatamente um recurso e sempre que houver fabricação de uma peça $i \in \mathcal{I}$, em um novo período, haverá gasto com setup. A restrição (3-7) garante que o custo de setup será contabilizado caso haja produção da peça $i \in \mathcal{I}$ no período $t \in \mathcal{T}$. Por fim, as restrições (3-8) e (3-9) garantem não-negatividade das variáveis e a definição de $\gamma_{i, t}$ como variável binária para toda peça $i \in \mathcal{I}$ e período $t \in \mathcal{T}$. Após a definição do planejamento da produção dada pela solução do modelo (3-1)-(3-9), o pedido de peças é calculado conforme o processo apresentado no algorítimo 1

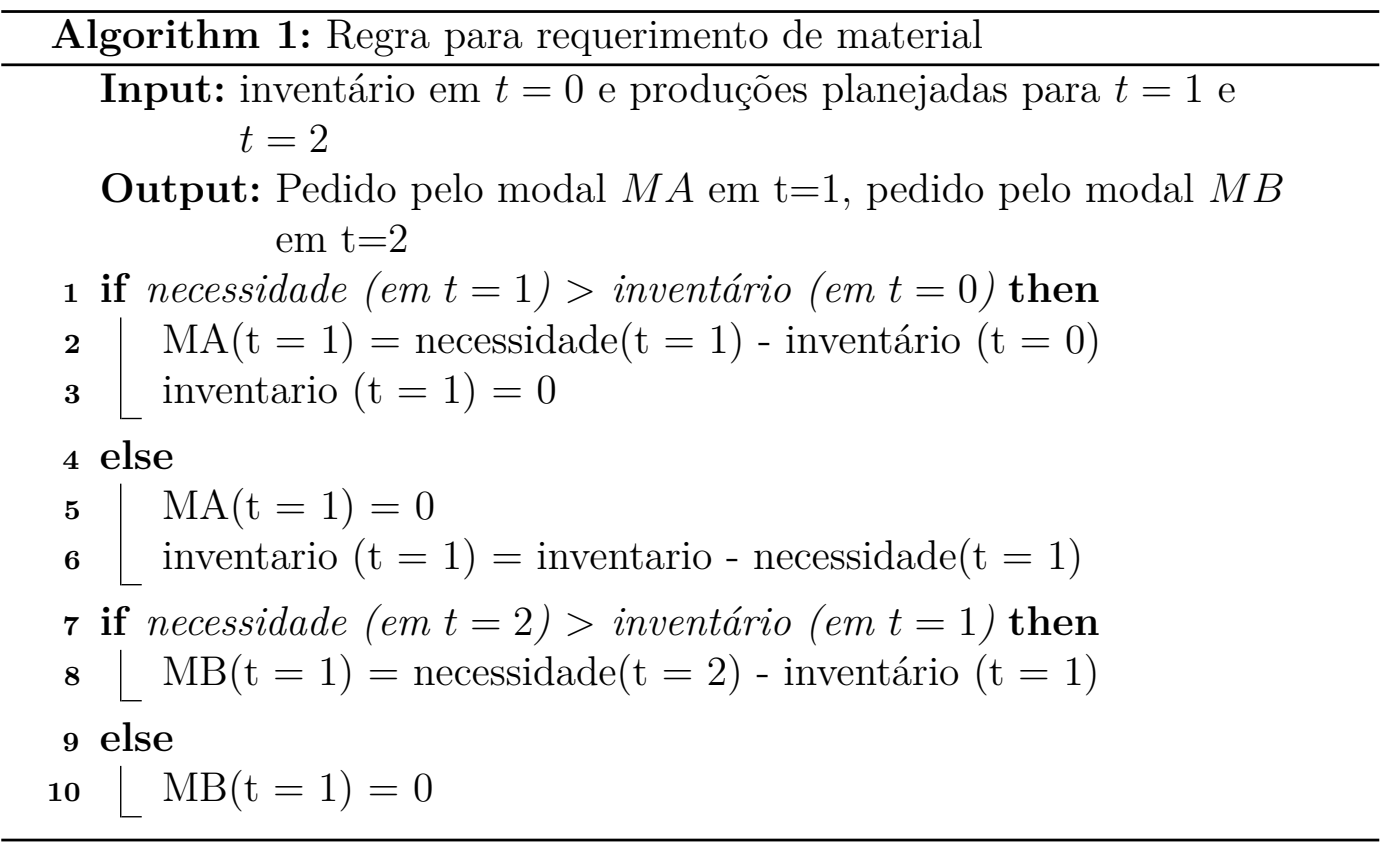


Capítulo 3. Planejamento Integrado de Produção e Compra sob Incerteza na

\section{2}

\section{Modelo Estocástico em Dois Estágios para o Planejamento da Produção com Requerimento Integrado de Material}

O modelo determinístico apresentado na seção 3.1 pode induzir a uma inconsistência no planejamento. Para mitigar esta inconsistência e realizar um melhor planejamento da produção, um modelo estocástico é proposto. Além disso, o requerimento de material é integrado ao modelo, possibilitando uma co-otimização da produção com as decisões de quantidade, momento de compra e modal de transporte. O modelo proposto é apresentado nas equações (3-10)(3-23) a seguir.

$\underset{\gamma_{i t \omega}, Q_{i t \omega k}, Y_{i t \omega}, B_{i t \omega}, X_{i t \omega}^{a}, X_{i t \omega}^{b}}{\operatorname{Minimize}} \sum_{\omega \in \Omega} P_{\omega} R_{\omega}$

subject to:

$$
\begin{aligned}
& R_{\omega}=\sum_{t \in \mathcal{T}} \sum_{i \in \mathcal{I}}\left(c_{i}^{s} \gamma_{i, t, \omega}+c_{i}^{e} Y_{i, t, \omega}+c_{i}^{a} X_{i, t, \omega}^{a}+c_{i}^{b} X_{i, t, \omega}^{b}+\sum_{k \in K} c_{i, k}^{p} Q_{i, t, \omega, k}+c_{i}^{d} B_{i, t, \omega}\right), \\
& \forall \omega \in \Omega ; \\
& Y_{i}^{0}+\sum_{k \in K} Q_{i, t, k}+X_{i, t}^{a}-\sum_{j \in s_{i}} \alpha_{i, j}\left(\sum_{k \in K} Q_{j, t, k}\right)-Y_{i, t, \omega}+B_{i, t, \omega}=d_{i, t, \omega}, \\
& \forall i \in \mathcal{I}, t \in \mathcal{T} \mid t=1, \omega \in \Omega \\
& Y_{i, t-1, \omega}+\sum_{k \in K} Q_{i, t, \omega, k}+X_{i, t, \omega}^{a}+X_{i, t-1}^{b}-\sum_{j \in s_{i}} \alpha_{i, j}\left(\sum_{k \in K} Q_{j, t, \omega, k}\right)-Y_{i, t, \omega} \\
& +B_{i, t, \omega}=d_{i, t, \omega}, \quad \forall i \in \mathcal{I}, t \in \mathcal{T} \mid t=2, \omega \in \Omega ; \\
& Y_{i, t-1, \omega}+\sum_{k \in K} Q_{i, t, \omega, k}+X_{i, t, \omega}^{a}+X_{i, t-1, \omega}^{b}-\sum_{j \in s_{i}} \alpha_{i, j}\left(\sum_{k \in K} Q_{j, t, \omega, k}\right)-Y_{i, t, \omega} \\
& +B_{i, t, \omega}=d_{i, t, \omega}, \quad \forall i \in \mathcal{I}, t \in \mathcal{T} \mid t \notin\{1,2\}, \omega \in \Omega ;(3-14) \\
& Y_{i}^{0}+Q_{i, t, 1}+X_{i, t}^{a}-\sum_{j \in s_{i}} \alpha_{i, j} Q_{j, t, 1} \geq 0 \\
& \forall i \in \mathcal{I}, t \in \mathcal{T} \mid t=1, \omega \in \Omega ; \\
& Y_{i, t-1, \omega}+Q_{i, t, 1}+X_{i, t, \omega}^{a}+X_{i, t-1}^{b}-\sum_{j \in s_{i}} \alpha_{i, j} Q_{j, t, 1} \geq 0 \\
& \forall i \in \mathcal{I}, t \in \mathcal{T} \mid t=2, \omega \in \Omega \text {; } \\
& Y_{i, t-1, \omega}+Q_{i, t, 1}+X_{i, t, \omega}^{a}+X_{i, t-1, \omega}^{b}-\sum_{j \in s_{i}} \alpha_{i, j} Q_{j, t, 1} \geq 0 \\
& \forall i \in \mathcal{I}, t \in \mathcal{T} \mid t \notin\{1,2\}, \omega \in \Omega \\
& \sum_{j \in \mathcal{J}_{m}}\left(\tau_{j}^{p} Q_{j, t, \omega, k}+\tau_{j, k}^{s} \gamma_{j, t, \omega}\right) \leq \tau_{m, k}^{m}, \quad \forall m \in \mathcal{M}, t \in \mathcal{T}, \omega \in \Omega, k \in \mathcal{K} ; \\
& \sum_{k \in K} Q_{i, t, \omega, k} \leq \bar{Q}_{i} \gamma_{i, t, \omega}, \quad \forall i \in \mathcal{I}, t \in \mathcal{T}, \omega \in \Omega
\end{aligned}
$$




$$
\begin{array}{ll}
X_{i, t, \omega}^{b} \leq \bar{X}_{i}^{b}, & \forall i \in \mathcal{I}, t \in \mathcal{T}, \omega \in \Omega ; \\
X_{i, t, \omega}^{a} \leq \bar{X}_{i}^{a}, & \forall i \in \mathcal{I}, t \in \mathcal{T}, \omega \in \Omega ; \\
Q_{i, t, \omega, k}, Y_{i, t, \omega}, X_{i, t, \omega}^{a}, X_{i, t, \omega}^{b}, B_{i, t, \omega} \geq 0, & \forall i \in \mathcal{I}, t \in \mathcal{T}, \omega \in \Omega ; \\
\gamma_{i, t, \omega} \in\{0,1\}, & \forall i \in \mathcal{I}, t \in \mathcal{T}, \omega \in \Omega .
\end{array}
$$

O problema de otimização (3-10)-(3-23) é um modelo de programação (estocástica) linear inteiro-misto que busca minimizar o valor esperado do custo total (equação (3-10)), sendo que a restrição (3-11) define o custo total em cada cenário como a soma dos custos setup, estoque, pedido pelos modais "MA" e "MB", produção e falta para todos os itens em todos os períodos. As restrições (3-12), (3-13) e (3-14) garantem o balanceamento da produção, sem possibilidade de atraso. Caso a demanda de algum item dentro de um período não seja atendida, é contabilizada a falta do produto. É importante ressaltar que as variáveis de produção e as variáveis de compra, tanto por modal "MA" quanto "MB", no primeiro período são variáveis de estado e, portanto, decididas antes da realização do cenário de demanda. As restrições (3-15), (3-16) e (3-17) foram necessárias apenas pelo fato de estarmos considerando mais de uma faixa de produção $k \in \mathcal{K}$, elas garantem a não invertibilidade da ordem de produção de peças que possuem relação de precedência. A restrição (3-18) garante, por período, que a capacidade de cada recurso (que pode ser entendido como uma máquina, ou estação de trabalho) não será excedida. Considera-se que cada peça é fabricada utilizando-se de exatamente um recurso e que toda vez que houver fabricação de uma peça $i \in \mathcal{I}$, em um novo período $t \in \mathcal{T}$, haverá gasto com setup. A restrição (3-19) busca garantir que o custo de setup será contabilizado caso haja produção da peça $i \in \mathcal{I}$ no período $t \in \mathcal{T}$ e as retrições (3-20) e (3-21) garantem que a máxima capacidade de compra não seja excedida em nenhum período e cenário. Por fim, nas restrição (3-22) e (3-23), temos a não-negatividade das variáveis e a definição de $\gamma_{i, t, \omega}$ como variável binária $\forall i \in \mathcal{I}, t \in \mathcal{T}, \omega \in \Omega$.

\section{3}

\section{Desenho do experimento}

\subsection{1}

\section{Dados de entrada}

Para avaliar os benefícios da metodologia de planejamento estocástico da produção co-otimizada com o requerimento de materiais, proposto na Seção (3.2), sob a prática usualmente implementada na indústria, que segue o 
procedimento descrito na Seção (3.1), um experimento numérico foi desenhado com dados realistas de uma fábrica automotiva. No estudo, foi considerada a produção de um único produto fabricado a partir de 6 materiais, com consumos de $5,5,1,2,1$, e 2 itens por produto. Foi considerada apenas uma estação $(|\mathcal{M}|=1)$ cujo tempo de produção atribuído foi de 1 hora por produto, dividiuse a produção em 2 faixas $(|\mathcal{K}|=2)$ de capacidades 176 e 88 horas por mês. Essas faixas podem ser interpretadas como capacidade de produção em período de trabalho normal e capacidade de produção em hora-extra.

Não foi considerado um limite na quantidade produzida por dia de forma direta, apenas no tempo de produção. Da mesma forma, a compra de matériaprima não foi limitada. Foram considerados custos de produção $\left(c^{p}\right)$ unitários de $\mathrm{R} \$ 100,00$ e $\mathrm{R} \$ 200,00$ para cada faixa $(k \in \mathcal{K})$ respectivamente. O custo de inventário unitário $\left(c^{e}\right)$ atribuído para o produto final foi de $\mathrm{R} \$ 10,00$ e para matérias-primas $\mathrm{R} \$ 0,50$. O custo de pedido de matéria-prima, pelo modal "MA" $\left(c^{a}\right)$ e "MB" $\left(c^{b}\right)$, foi de $\mathrm{R} \$ 10,00$ e $\mathrm{R} \$ 4,00$, respectivamente. O custo de deficit $\left(c^{d}\right)$ de qualquer peça ou do produto final foi considerado como sendo $\mathrm{R} \$ 1000,00$.

Não foi considerado lead time de produção $\left(l^{p}\right)$ fora o tempo de produção de uma hora. Foi considerado um lead time de pedido pelo modal "MA" $\left(l^{a}\right)$ de um mês e um lead time de pedido pelo modal "MB" $\left(l^{b}\right)$ de dois meses. Para o inventário inicial, foi considerado que de produtos finais haviam exatamente a previsão de demanda do primeiro período, além de peças suficientes para se produzirem mais 100 produtos. Por termos a produção de apenas um produto, não foi considerado tempo e custo de setup. A previsão da demanda é explicada na próxima seção.

\subsection{2}

\section{Previsão e Simulação dos Cenários de Demanda}

Este experimento numérico considerou apenas um produto final, cujo histórico de demanda foi baseado em uma série de vendas de veículos do Ipeadata, conforme Figura 3.1. Considerou-se a série do Ipeadata dividida por $10 \mathrm{E} 3$.

Por um lado, para o modelo determinístico, a previsão da demanda foi definida a partir da predição 12 períodos a frente utilizando a função auto . arima do Software R. Por outro lado, a geração dos cenários de demanda para o modelo estocástico também se utilizou da função auto.arima do Software R com uma metodologia de simulação baseada em bootstrap para os resíduos. 


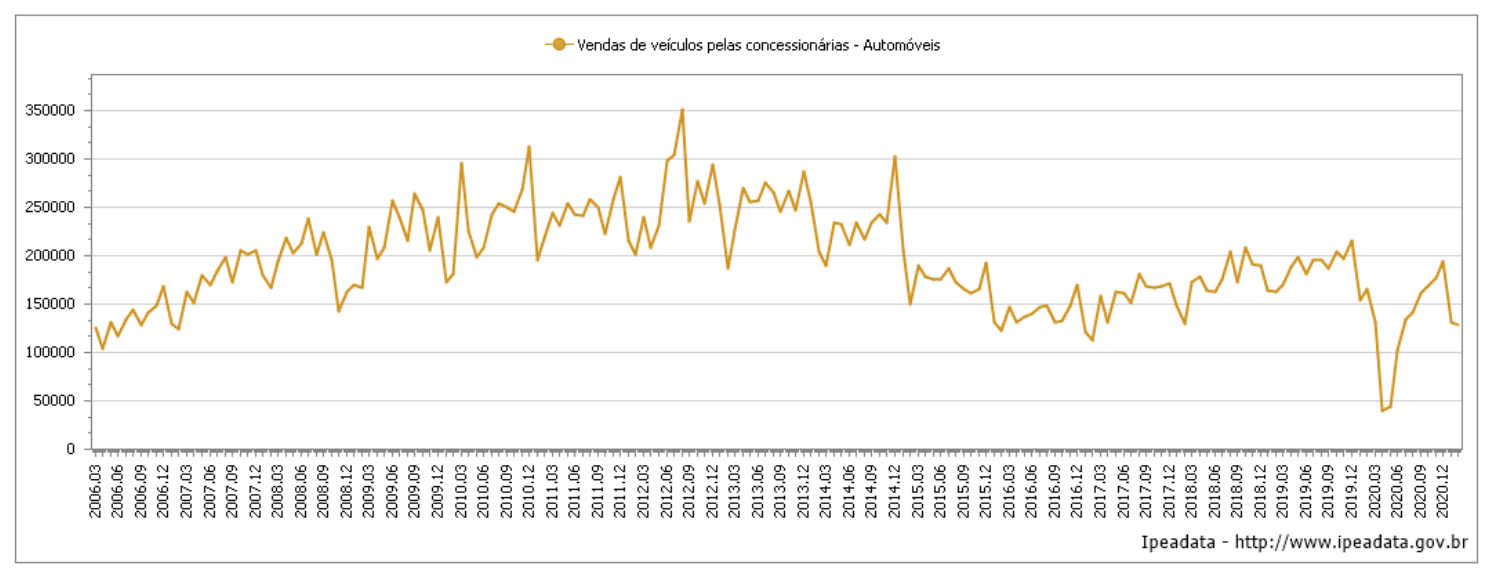

Figura 3.1: Série histórica de venda de veículos do Ipeadata.

Dado o modelo gerado pela função auto.arima, a metodologia de bootstrap nos resíduos, permite a simulação de um conjunto completo de valores futuros para a série temporal, assumindo que os resíduos do futuro serão semelhantes aos resíduos do passado. Veja (Hyndman, 2018) para mais detalhes.

Devido a forte alteração na demanda provocada pelo COVID em 2020 optamos por não utilizar os dados desse ano. Como foi considerado um planejamento anual com horizonte rolante mensal, para não entrar no planejamento de 2020 optamos por usar os dados até 2017 para ajuste de metodologia e a realização de 2018 para avaliação dos resultados.

\subsection{3}

\section{Algoritmo de comparação}

As duas metodologias foram comparadas considerando um planejamento anual com horizonte rolante mensal, congelamento de produção dentro do mês e lead time de dois meses para o pedido por modal "MB" e lead time de um mês para o pedido por modal "MA". Para realizar a comparação das duas metodologias foi observado os custos planejados e reais que cada um dos modelos de planejamento/operação incorreriam por mês no decorrer de um ano. O algorítimo elaborado para a comparação está descrito no algoritmo 2, onde " $N$ " representa o número de simulações que devem ser realizadas.

O algoritmo descrito foi implementado em linguagem Julia. Os modelos de planejamento apresentados no capítulo foram resolvidos usando Gurobi Solver 9.0 sob a codificação em JuMP. Todos resultados foram obtidos em uma máquina Intel Core i7-10700K 4.8GHz com 64 GB de RAM. 


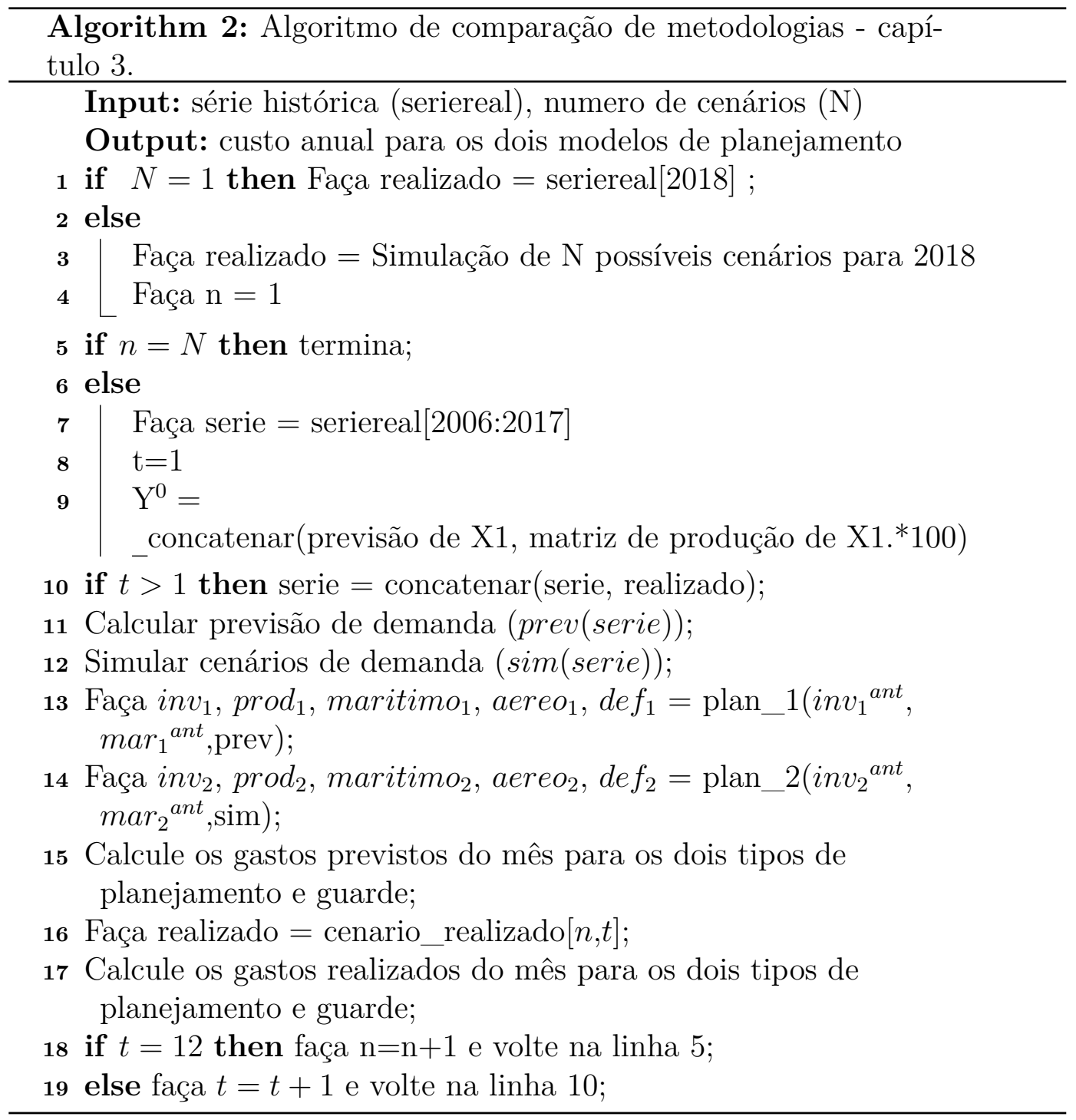

\section{4}

\section{Resultados Numéricos}

Nesta seção, iremos apresentar os resultados numéricos do experimento desenhado na Seção 3.3 comparando o modelo estocástico proposto neste trabalho com a metodologia tipicamente utilizada na indústria. Iremos avaliar três dimensões principais: (i) uma análise out-of-sample, avaliando o impacto financeiro no planejamento da produção seguindo os dois modelos através da demanda real observada para o ano de 2018; (ii) uma análise probabilística da performance dos modelos a partir de um novo conjunto de cenários simulados de acordo com a seção 3.3.2; e (iii) uma análise de sensibilidade dos parâmetros do problema nos modelos estudados. Para as dimensões (i) e (ii) considerouse 1000 cenários de demanda para o modelo estocástico, para a dimensão (iii) considerou-se 100 cenários. Nas análises que seguem, iremos nos referenciar ao modelos determinístico como M.01 e ao modelo estocástico como M.02. 


\subsection{1}

\section{Análise Baseada na Demanda Real Observada para 2018}

Utilizando o modelo determinístico apresentado em (3-1)-(3-9) para o planejamento da produção do ano de 2018 obtivemos um custo total de $\mathrm{R} \$$ 494.815,00, enquanto que utilizando o modelo estocástico proposto (3-10)(3-23), o custo esperado para o ano foi de $\mathrm{R} \$ 460.000,110$, resultando em uma economia (percentual) de aproximadamente 7\%. Com relação a aderência entre a política planejada e a efetivamente implementada (realizada), enquanto que no modelo determinístico o custo total realizado foi $16,26 \%$ maior do que o planejado, para o modelo estocástico, o acréscimo no custo foi inferior a $1 \%$.

A Figura 3.2 apresenta (mês-a-mês) a diferença entre custo total planejado e o efetivamente observado usando os dados reais para 2018 para cada um dos modelos. Analogamente, na Figura 3.3 são apresentados individualmente cada parcela do custo total, comparando o planejado com o efetivamente observado usando os dados reais para 2018.

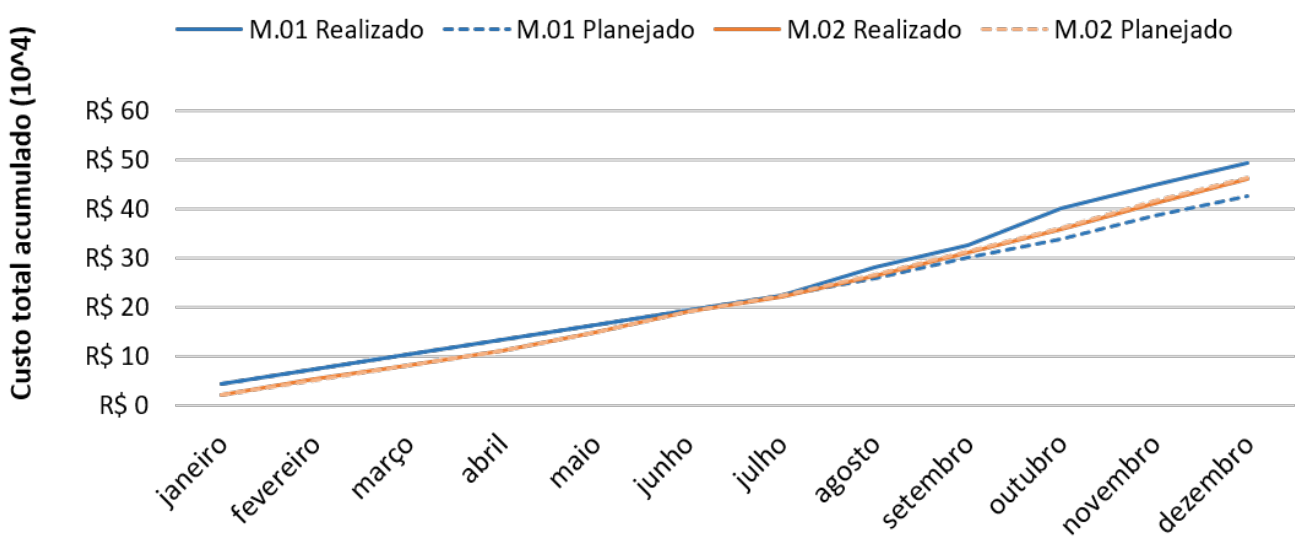

Figura 3.2: Custo total planejado e o efetivamente observado usando os dados reais para 2018 para cada um dos modelos analisados.

Observando os gráficos da Figura 3.3, por parcela de custo, vemos que o modelo proposto (M.02), com excessão do primeiro mês, apresenta um maior nível de produção, um volume superior de compra por modo "MB" e, a partir de maio, um volume maior de inventário. Tais observações explicam um custo déficit zerado no M.02 em comparação a um custo de $\mathrm{R} \$ 69.00,00$ no M.01. Como o custo de deficit é muito superior aos outros custos, vemos que o modelo proposto busca mitigar a exposição a este custo aumentando a produção e, conjuntamente, realizando mais pedidos por meio do modal "MB". A exceção na produção do primeiro mês pode ser explicada pelo nível 
de estoque considerada no início da operação. Devido ao alto custo de pedido pelo modal "MA", como apenas o M.02 observa os custos de pedido, este prefere utilizar apenas a capacidade produtiva referente ao nível de estoque do período. O M.02 deixa, portanto, de se beneficiar do preço da produção inicialmente, uma vez que deixa de produzir ao nível mais barato $(k=1)$, visando minimizar o custo de pedido por todo o horizonte de análise. Tal comportamento também explica o motivo do custo de inventário do M.01 ser superior nos primeiros 5 meses.

Como esperado, tais resultados nos mostram a inconsistência do modelo determinístico, uma vez que o modelo fica cego para os piores cenários, e só se prepara para o resultado médio, o que em nosso estudo de caso aumentou o deficit e consequentemente os custos. Além disso, a não otimização conjunta das variáveis de compra no M.01 fez com que esse modelo perdesse a oportunidade de alterar a produção buscando minimizar os custos de pedido, como foi observado nos primeiros períodos do M.02.

\subsection{2}

\section{Análise Probabilística para 2018}

A fim de realizar uma análise probabilística da performance dos modelos de planejamento determinístico (M.01) e estocástico (M.02), um novo conjunto de 5000 cenários foi simulado. De maneira geral, foi observado que, em média, há uma redução da ordem de $3,57 \%$ no custo total ao se utilizar o modelo proposto com relação ao determinístico. Na Figura 3.4, a distribuição acumulada (amostral) da diferença percentual entre o custo total do modelo estocástico e determinístico é apresentada.

O resultado apresentado no gráfico 3.4 nos indica que em $75 \%$ dos casos houve uma diminuição dos custos com o modelo M.02. Além disso, nos $10 \%$ dos casos onde há maior vantagem em se utilizar modelo M.01, a perda com o modelo M.02 é em média de 5\%, enquanto que nos $10 \%$ dos casos onde há maior vantagem em se utilizar o modelo M.02, a perda com o modelo $M .01$ é em média de $14 \%$. Tais resultados confirmam mais uma vez a vantagem da utilização do modelo M.02 em relação ao modelo M.01.

\subsection{3}

\section{Análise de sensibilidade}

Nessa seção, iremos realizar uma análise de sensibilidade dos parâmetros críticos do problema, avaliando o impacto (percentual) nos custos totais 


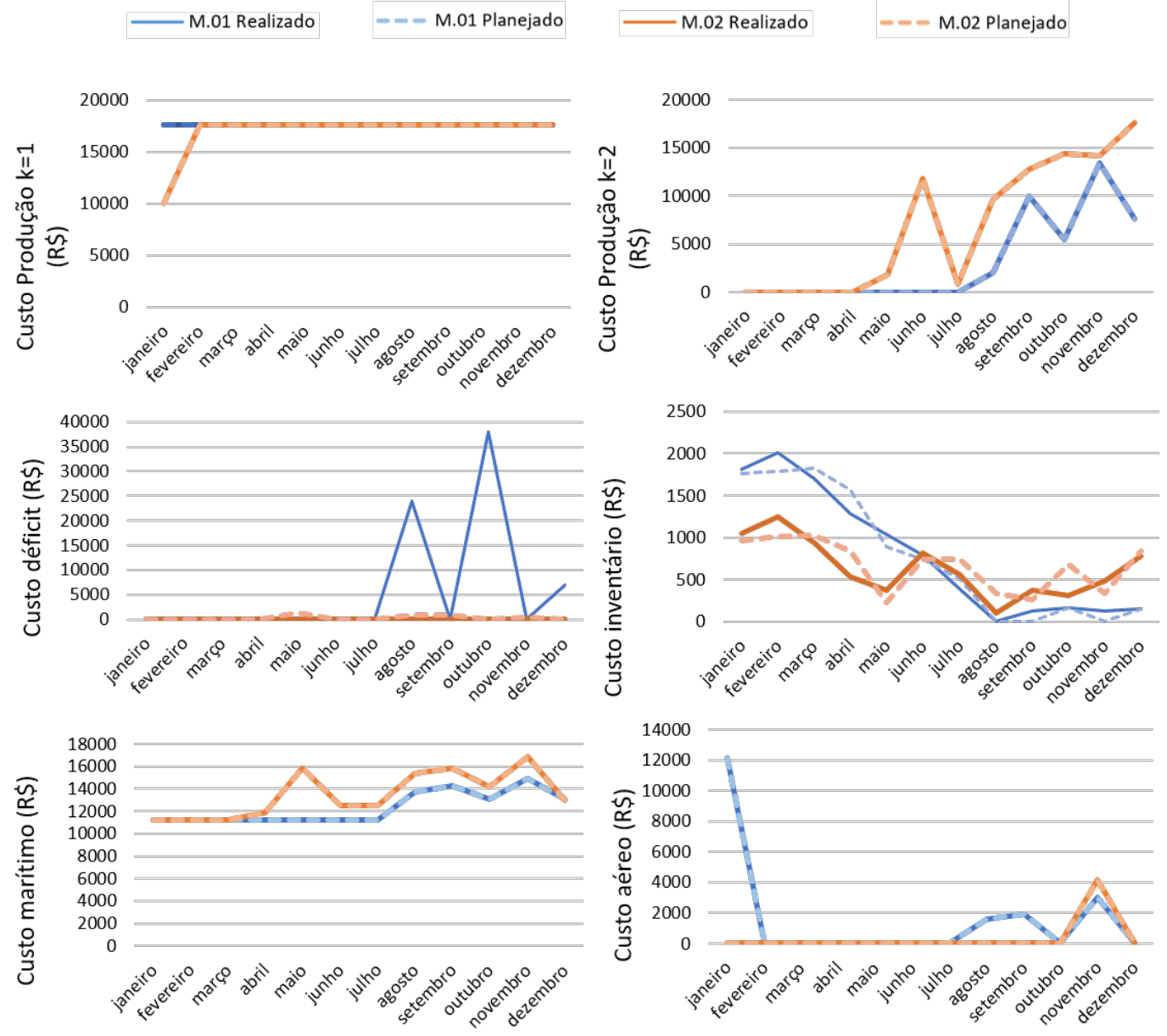

Figura 3.3: Parcelas do custo total comparando o planejado com o efetivamente observado usando os dados reais para 2018 entre cada modelo analisado.

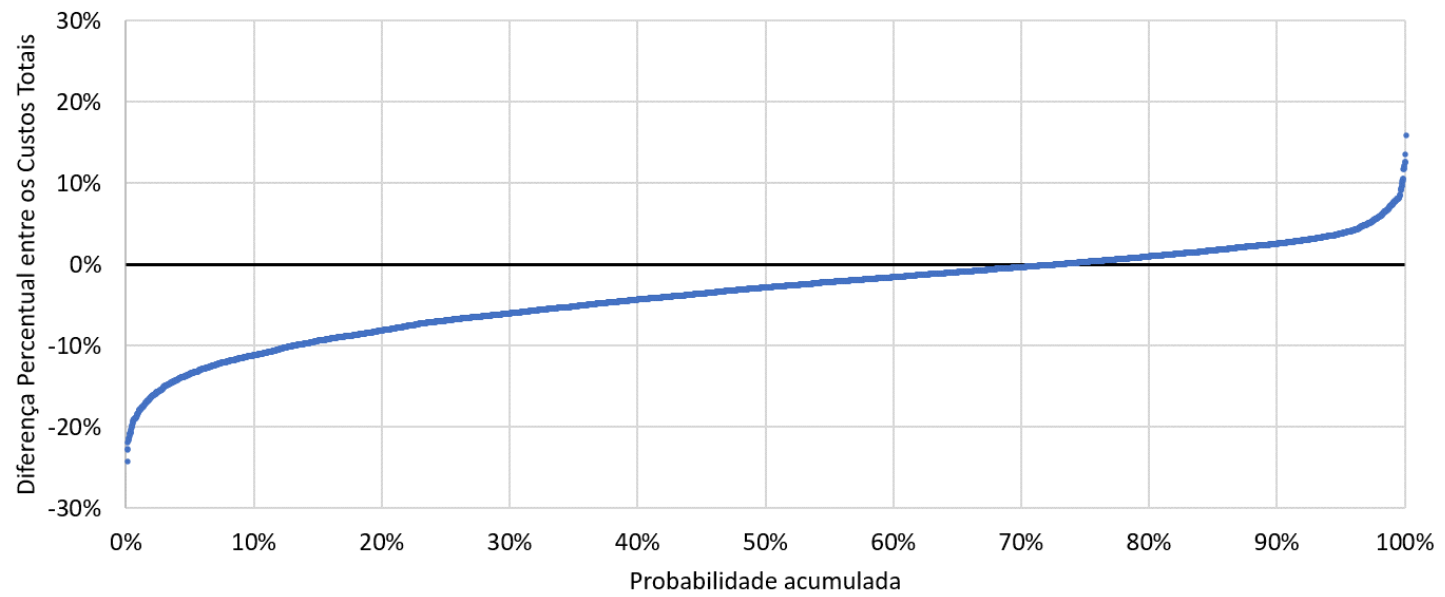

Figura 3.4: Distribuição acumulada (amostral) da diferença percentual entre o custo total do modelo estocástico e determinístico 
incorridos pelo modelo M.02 em relação ao modelo M.01. Foram consideradas 72 configurações, geradas pela combinação de 4 valores para custo de falta $\left(c^{f}\right)$, 3 valores para custo de produção na faixa $k=2\left(c^{p}\right), 3$ valores para custo de pedido pelo modal "MA" $\left(c^{a}\right)$ e 2 tempos de produção unitário $\left(\tau^{p}\right)$. A figura 3.5 ilustra o impacto de cada configuração avaliada.

Primeiramente, há um significativo aumento (relativo) do custo do modelo determinístico a medida que o custo de falta aumenta. Esta observação está em linha com os resultados observados na Seção 3.4.1 em que o modelo determinístico não é capaz de valorar o impacto da falta de maneira adequada, induzindo a um alto custo associado. Além disso, o tempo de produção também possui um alto impacto no planejamento advindo dos modelos analisados neste trabalho. Mais especificamente, uma diminuição no tempo de produção induz a um aumento relativo da diferença do custo do modelo M.01 em comparação ao modelo M.02. Intuitivamente, podemos entender que quanto menor o tempo de produção, maior o tempo disponível para produção, o que diminui a necessidade de estoque, principalmente para o modelo M.01 que entende que pode produzir a sua produção no próprio período de demanda, quando a demanda é maior do que o esperado o modelo acaba por cair em um alto custo de deficit, o que não ocorre tanto com o modelo M.02 que já estava visualizando os possíveis cenários. Por fim, observamos que, no experimento analisado neste trabalho, o impacto relativo do custo de produção na faixa $k=2$ e custo de pedido pelo modal "MA" não é significativo entre os modelos de planejamento M.01 e M.02. 
Capítulo 3. Planejamento Integrado de Produção e Compra sob Incerteza na Demanda

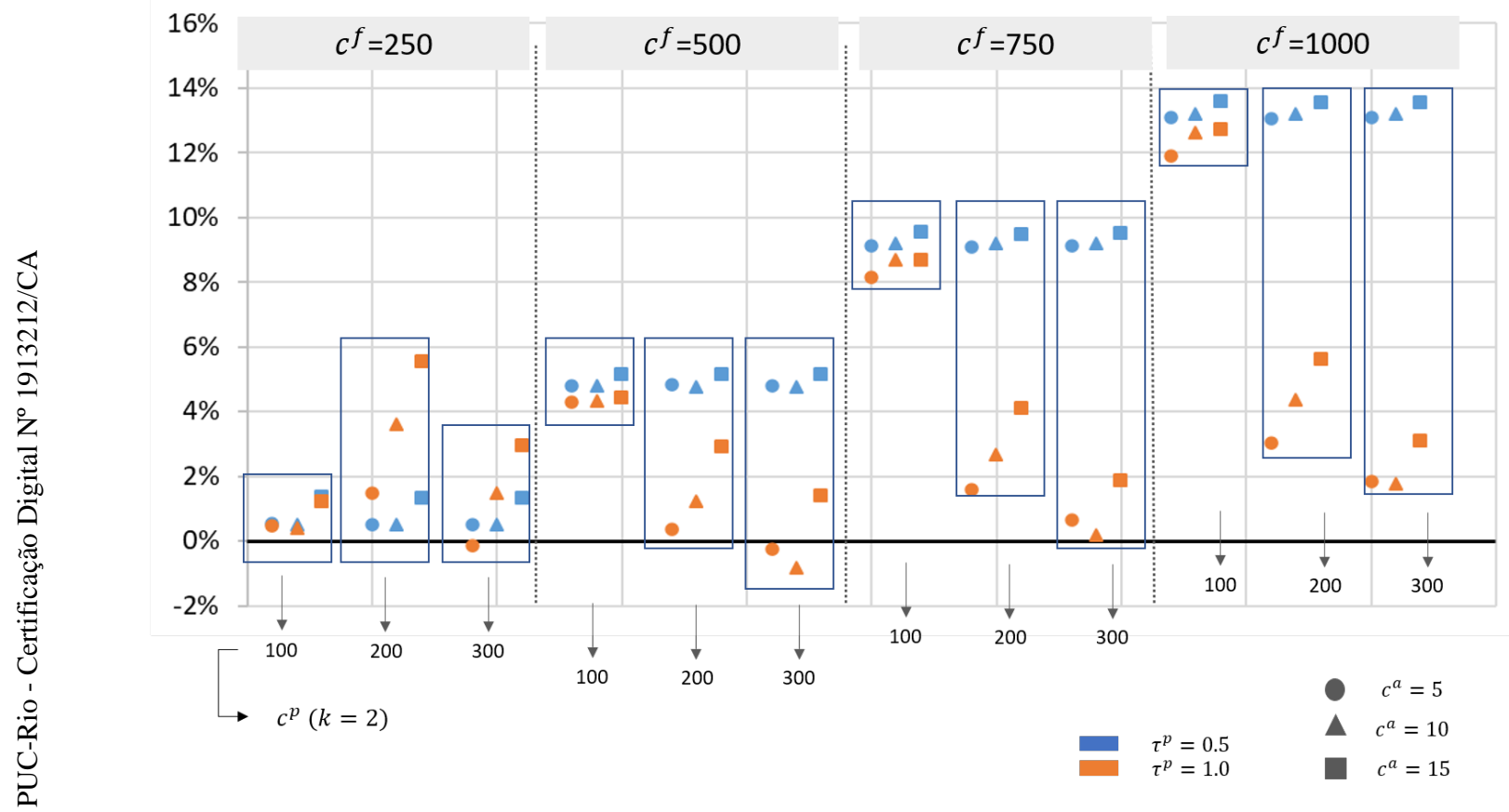

Figura 3.5: Diferença média por configuração dos parâmetros 


\section{Planejamento Integrado de Produção, Compra e Sequencia- mento de jobs, sob Incerteza na Demanda}

Até agora vimos o impacto nos custos da produção por se considerar a demanda de forma determinística e por se determinar o pedido de material apenas após a solução do planejamento de produção. Nesse capítulo buscamos ter uma ideia a respeito dos problemas que podem ocorrer ao não se considerar as restrições de sequenciamento de produção na hora de se determinar o plano mestre da produção.

O capítulo está organizado da seguinte forma: Nas duas primeiras seções iremos detalhar um modelo e uma heurística que buscam incluir as restrições de sequenciamento ao modelo da seção 3.2. Na seção 4.3 apresentaremos o desenho do experimento, que foi alterado devido a natureza da análise aqui proposta. Na seção 4.4 os resultados numéricos serão apresentamos.

Para uma melhor compreensão dos modelos apresentados nesta capítulo, na Tabela 4.1, é apresentada uma lista de nomenclatura contendo os conjuntos, parâmetros e variáveis utilizados. Por simplicidade, neste trabalho, foi considerado um período unitário para o lead time de pedido pelo modal flexível "MA", de dois períodos para o pedido pelo modal de baixo custo "MB" e nulo para o lead time de produção.

\begin{tabular}{ll}
\hline Conjuntos & \\
$\mathcal{I}$ & Conjunto de peças \\
$\mathcal{I}_{q}$ & Conjunto de peças que podem ser produzidas internamente \\
$\mathcal{T}$ & Conjunto de períodos \\
$\mathcal{K}$ & Conjunto de faixas de produção \\
$\mathcal{M}$ & Conjunto de máquinas/estações de trabalho \\
$\mathcal{N}(k)$ & Conjunto de intervalos de sequenciamento \\
& na faixa de produção $k \in \mathcal{K}$ \\
$\Omega$ & Conjunto de cenários de demanda \\
$\mathcal{J}_{m}$ & Conjunto de peças $i \in \mathcal{I}$ produzidas com o recurso $m \in \mathcal{M}$ \\
$\mathcal{M}_{j}$ & Conjunto de recursos $m \in \mathcal{M}$ que produzem a peça $j \in \mathcal{I}_{q}$ \\
$S_{i}$ & Conjunto de peças sucessoras diretas da peça $i \in \mathcal{I}$ \\
$S_{j}$ & Conjunto de peças sucessoras da peça $j \in \mathcal{I}_{q}$ \\
\hline
\end{tabular}




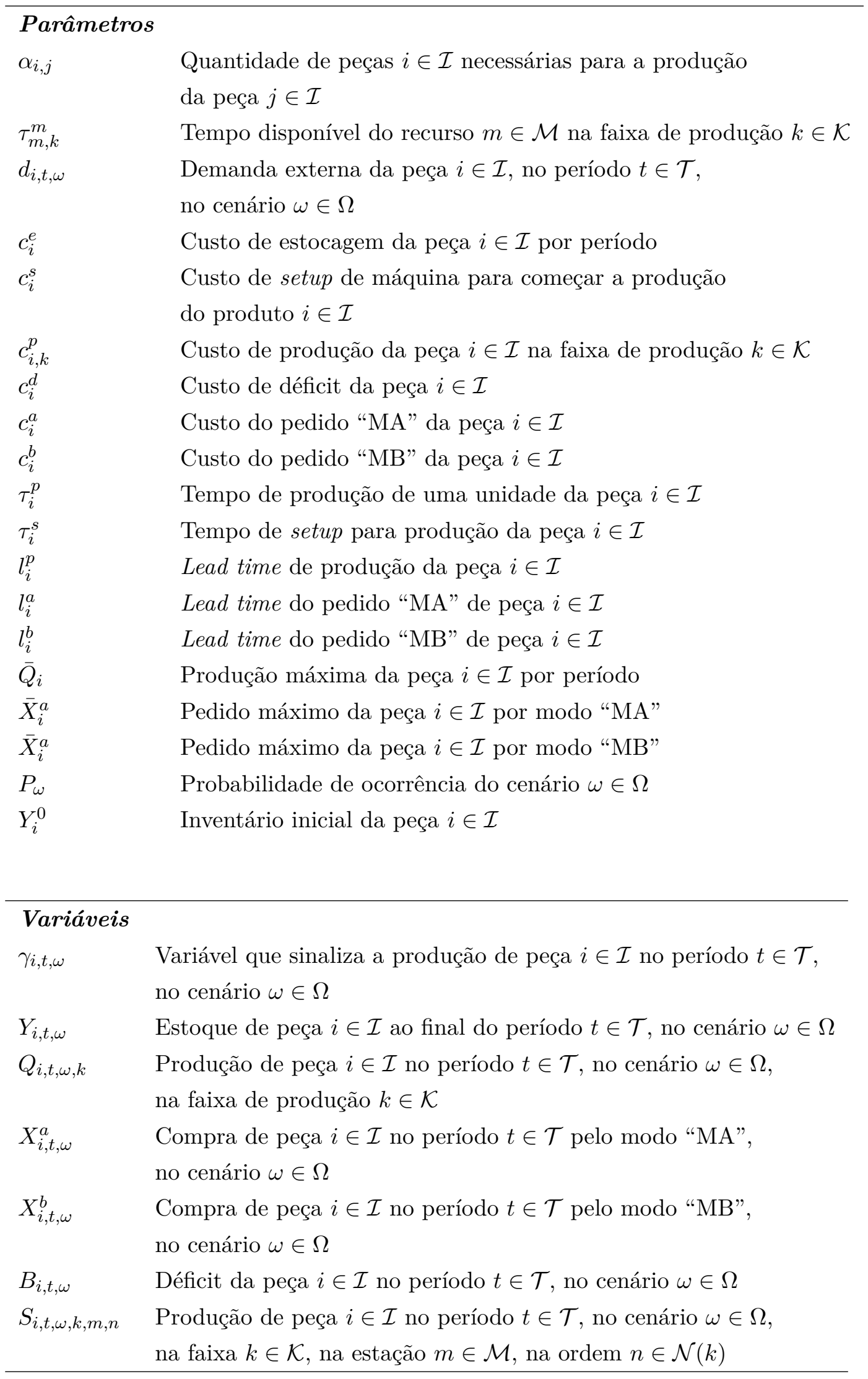

Tabela 4.1: Lista com a nomenclatura contendo os conjuntos, parâmetros e variáveis utilizados. 
Capítulo 4. Planejamento Integrado de Produção, Compra e Sequenciamento de jobs, sob Incerteza na Demanda

\section{1}

\section{Modelo Estocástico em Dois Estágios para o Planejamento da Produção com Requerimento Integrado de Material e Viabilidade do Sequencia- mento da Produção}

Tanto o modelo determinístico apresentado na seção 3.1, quanto o modelo proposto na seção 3.2 podem induzir a uma inconsistência no planejamento devido a toda a "restrição de sequenciamento" estar contida nas restrições (3-6) e (3-18), respectivamente. Basicamente, nesses modelos está sendo considerado apenas um tempo fixo de produção para cada peça em cada máquina e, embora haja uma restrição de balanço da produção em todos os períodos, os modelos não admitem que possa haver a necessidade de um tempo de espera entre uma peça "x1" e uma peça "x2" que precisa da anterior para a sua fabricação.

Para mitigar esta inconsistência e realizar um melhor planejamento da produção, substituímos aqui a restrição (3-18) pelas restrições (4-9)-(4-12). O modelo proposto é apresentado nas equações (4-1)-(4-17) a seguir.

$$
\underset{\gamma_{i t \omega}, Q_{i t \omega k}, Y_{i t \omega}, B_{i t \omega}, X_{i t \omega}^{a}, X_{i t \omega}^{b}}{\operatorname{Minimiz}} \sum_{\omega \in \Omega} P_{\omega} R_{\omega}
$$

subject to:

$$
\begin{aligned}
& R_{\omega}=\sum_{t \in \mathcal{T}} \sum_{i \in \mathcal{I}}\left(c_{i}^{s} \gamma_{i, t, \omega}+c_{i}^{e} Y_{i, t, \omega}+c_{i}^{a} X_{i, t, \omega}^{a}+c_{i}^{b} X_{i, t, \omega}^{b}+\sum_{k \in K} c_{i, k}^{p} Q_{i, t, \omega, k}+c_{i}^{d} B_{i, t, \omega}\right), \\
& \forall \omega \in \Omega \\
& Y_{i}^{0}+\sum_{k \in K} Q_{i, t, k}+X_{i, t}^{a}-\sum_{j \in s_{i}} \alpha_{i, j}\left(\sum_{k \in K} Q_{j, t, k}\right)-Y_{i, t, \omega}+B_{i, t, \omega}=d_{i, t, \omega} \\
& \forall i \in \mathcal{I}, t \in \mathcal{T} \mid t=1, \omega \in \Omega ; \\
& Y_{i, t-1, \omega}+\sum_{k \in K} Q_{i, t, \omega, k}+X_{i, t, \omega}^{a}+X_{i, t-1}^{b}-\sum_{j \in s_{i}} \alpha_{i, j}\left(\sum_{k \in K} Q_{j, t, \omega, k}\right)-Y_{i, t, \omega} \\
& +B_{i, t, \omega}=d_{i, t, \omega}, \quad \forall i \in \mathcal{I}, t \in \mathcal{T} \mid t=2, \omega \in \Omega ; \\
& Y_{i, t-1, \omega}+\sum_{k \in K} Q_{i, t, \omega, k}+X_{i, t, \omega}^{a}+X_{i, t-1, \omega}^{b}-\sum_{j \in s_{i}} \alpha_{i, j}\left(\sum_{k \in K} Q_{j, t, \omega, k}\right)-Y_{i, t, \omega} \\
& +B_{i, t, \omega}=d_{i, t, \omega}, \quad \forall i \in \mathcal{I}, t \in \mathcal{T} \mid t \notin\{1,2\}, \omega \in \Omega \text {; } \\
& Y_{i}^{0}+Q_{i, t, 1}+X_{i, t}^{a}-\sum_{j \in s_{i}} \alpha_{i, j} Q_{j, t, 1} \geq 0 \\
& \forall i \in \mathcal{I}, t \in \mathcal{T} \mid t=1, \omega \in \Omega \text {; } \\
& Y_{i, t-1, \omega}+Q_{i, t, 1}+X_{i, t, \omega}^{a}+X_{i, t-1}^{b}-\sum_{j \in s_{i}} \alpha_{i, j} Q_{j, t, 1} \geq 0 \\
& \forall i \in \mathcal{I}, t \in \mathcal{T} \mid t=2, \omega \in \Omega
\end{aligned}
$$




$$
\begin{aligned}
& Y_{i, t-1, \omega}+Q_{i, t, 1}+X_{i, t, \omega}^{a}+X_{i, t-1, \omega}^{b}-\sum_{j \in s_{i}} \alpha_{i, j} Q_{j, t, 1} \geq 0 \\
& \forall i \in \mathcal{I}, t \in \mathcal{T} \mid t \notin\{1,2\}, \omega \in \Omega \\
& Q_{i, t, \omega, k}=\sum_{m \in \mathcal{M}} \sum_{n \in \mathcal{N}[k]} S_{i, t, \omega, k, m, n}, \quad \forall i \in \mathcal{I}_{q}, t \in \mathcal{T}, \omega \in \Omega, k \in K \\
& \sum_{i \in \mathcal{I}_{q}} S_{i, t, \omega, k, m, n}<=1, \quad \forall t \in \mathcal{T}, \omega \in \Omega, k \in K, m \in \mathcal{M}, n \in \mathcal{N}[k] ;(4-10) \\
& \sum_{i \in \mathcal{I}_{m}} \sum_{n \in \mathcal{N}[k]}\left(\tau_{i}^{p} S_{i, t, \omega, k, m, n}+\tau_{i, k}^{s} \gamma_{i, t, \omega}\right) \leq \tau_{m, k}^{m} \\
& \forall t \in \mathcal{T}, \omega \in \Omega, k \in K, m \in \mathcal{M} \\
& \alpha_{j, i}\left(\sum_{m \in j M[i]} \sum_{n \in\left[1, n^{\prime}\right]} S_{i, t, \omega, k, m, n}+\sum_{m \in j M[i]} \sum_{c \in[1, k-1]} \sum_{n \in N[c]} S_{i, t, \omega, c, m, n}\right) \\
& \leq \sum_{m \in j M[j]} \sum_{n \in\left[1,\left(n^{\prime}-1\right)\right]} S_{j, t, \omega, k, m, n}+\sum_{m \in j M[j]} \sum_{c \in[1, k-1]} \sum_{n \in N[c]} S_{j, t, \omega, c, m, n}+Y_{j, t-1, \omega}, \\
& \forall j \in \mathcal{I}_{q}, i \in S_{j}, t \in \mathcal{T}, \omega \in \Omega, n^{\prime} \in N[k], k \in K ; \\
& \sum_{k \in K} Q_{i, t, \omega, k} \leq \bar{Q}_{i} \gamma_{i, t, \omega}, \quad \forall i \in \mathcal{I}, t \in \mathcal{T}, \omega \in \Omega ; \\
& X_{i, t, \omega}^{b} \leq \bar{X}_{i}^{b} \\
& \forall i \in \mathcal{I}, t \in \mathcal{T}, \omega \in \Omega ; \\
& X_{i, t, \omega}^{a} \leq \bar{X}_{i}^{a} \\
& \forall i \in \mathcal{I}, t \in \mathcal{T}, \omega \in \Omega \text {; } \\
& Q_{i, t, \omega, k}, Y_{i, t, \omega}, X_{i, t, \omega}^{a}, X_{i, t, \omega}^{b}, B_{i, t, \omega} \geq 0 \\
& \forall i \in \mathcal{I}, t \in \mathcal{T}, \omega \in \Omega \text {; } \\
& \gamma_{i, t, \omega}, S_{i, t, \omega, k, m, n} \in\{0,1\} \text {, } \\
& \forall i \in \mathcal{I}, t \in \mathcal{T}, \omega \in \Omega, k \in K, m \in \mathcal{M}, n \in \mathcal{N}[k] .
\end{aligned}
$$

O problema de otimização (4-1)-(4-16) é similar ao problema apresentado na seção 3.2 do capítulo 3. A diferença entre os modelos consiste na substituição da restrição (3-18) pelas restrições (4-9)-(4-12), por isso nos limitaremos a explicar as restrições (4-9)-(4-12) aqui.

A restrição (4-9) faz a ligação da produção total da peça $i \in \mathcal{I}$, no período $t \in \mathcal{T}$, no cenário $\omega \in \Omega$, na faixa de produção $k \in \mathcal{K}$ com a soma das unidades da mesma peça, produzidas no mesmo período, cenário e faixa de produção em cada recurso $m \in \mathcal{M}$ e intervalo $n \in \mathcal{N}[k]$. A restrição (4-10) garante que só seja planejado, no máximo, um produto por máquina $m \in \mathcal{M}$, por vez. A restrição (4-11), da mesma forma que a restrição (3-18) fazia, garante, por período, que a capacidade de cada recurso (que pode ser entendido como uma máquina, ou estação de trabalho) não será excedida. A diferença prática entre os modelos descritos nas seções3.2 e 4.1 encontra-se na restrição (4-12), onde busca-se definir que, em qualquer momento, a quantidade produzida de qualquer peça $i \in \mathcal{I}$ tem que ser tal que haja correspondência no número de peças $j \in \mathcal{I}_{q}$, necessárias a produção de $i$, no momento imediatamente anterior 
a este. A restrição (4-12) será exata quando o tempo de produção das peças for igual entre elas ou quando houver apenas uma máquina, caso contrário ela se torna uma aproximação para a restrição de balanço do sequenciamento. Para contornar essa aproximação, na próxima seção será apresentada uma heurística que garante a viabilidade do sequenciamento durante a etapa de planejamento.

\section{2}

\section{Heurística para Verificação da Viabilidade do Sequenciamento da Produ- ção Durante Etapa de Planejamento}

Devido as limitações do modelo apresentado na seção 4.1, nessa seção é analisada uma outra forma para se considerar o sequenciamento da produção ainda na fase de planejamento. A ideia é manter o modelo da seção 3.2 para realizar o planejamento, mas sempre que o modelo for executado, rodar um algoritmo que, através de um modelo de sequenciamento, verifica se o resultado é viável para o próximo mês. Caso a solução não seja viável a restrição 318 é "apertada", e o modelo de planejamento deve ser executado novamente. O processo é repetido até que o sequenciamento se torne viável. O processo é apresentado no algoritmo 3 e o modelo de sequenciamento é descrito nas restrições (4-18) - (4-25).

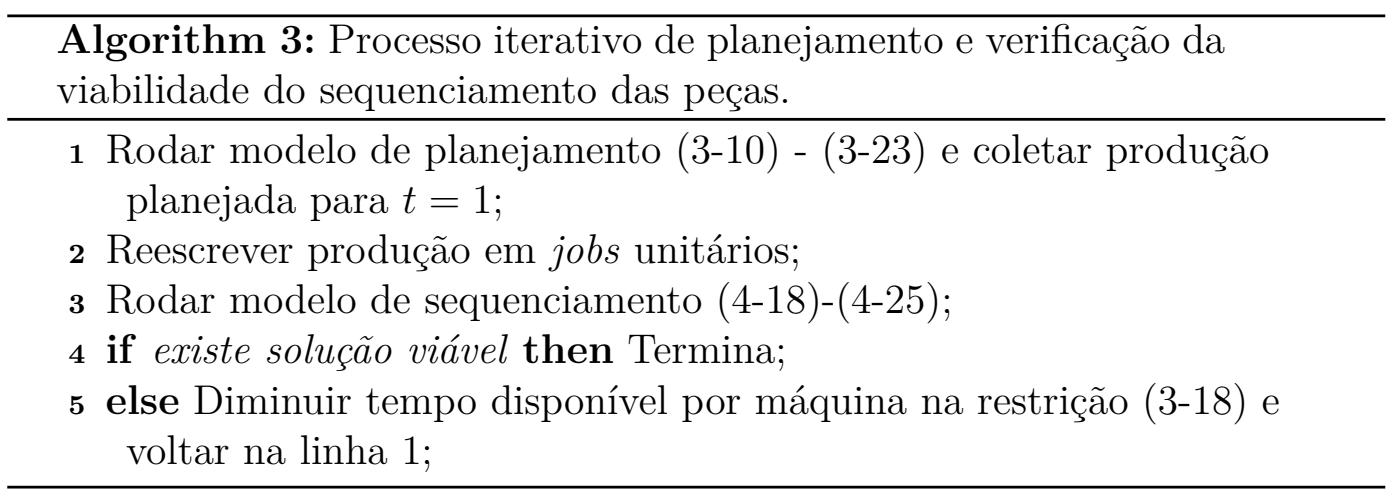

O modelo considerado para a verificação da viabilidade do sequenciamento das peças é similar ao descrito na seção 2.2 , a única diferença é que ao invés de minimizarmos o tempo de produção, estamos apenas interessados em verificar se foi possível a finalização dentro do período. A função de minimização do tempo $T$ 2-8 foi substituída pela restrição 4-25 que define que o tempo $T$ deve ser menor que o período total $\mathcal{T}$. 


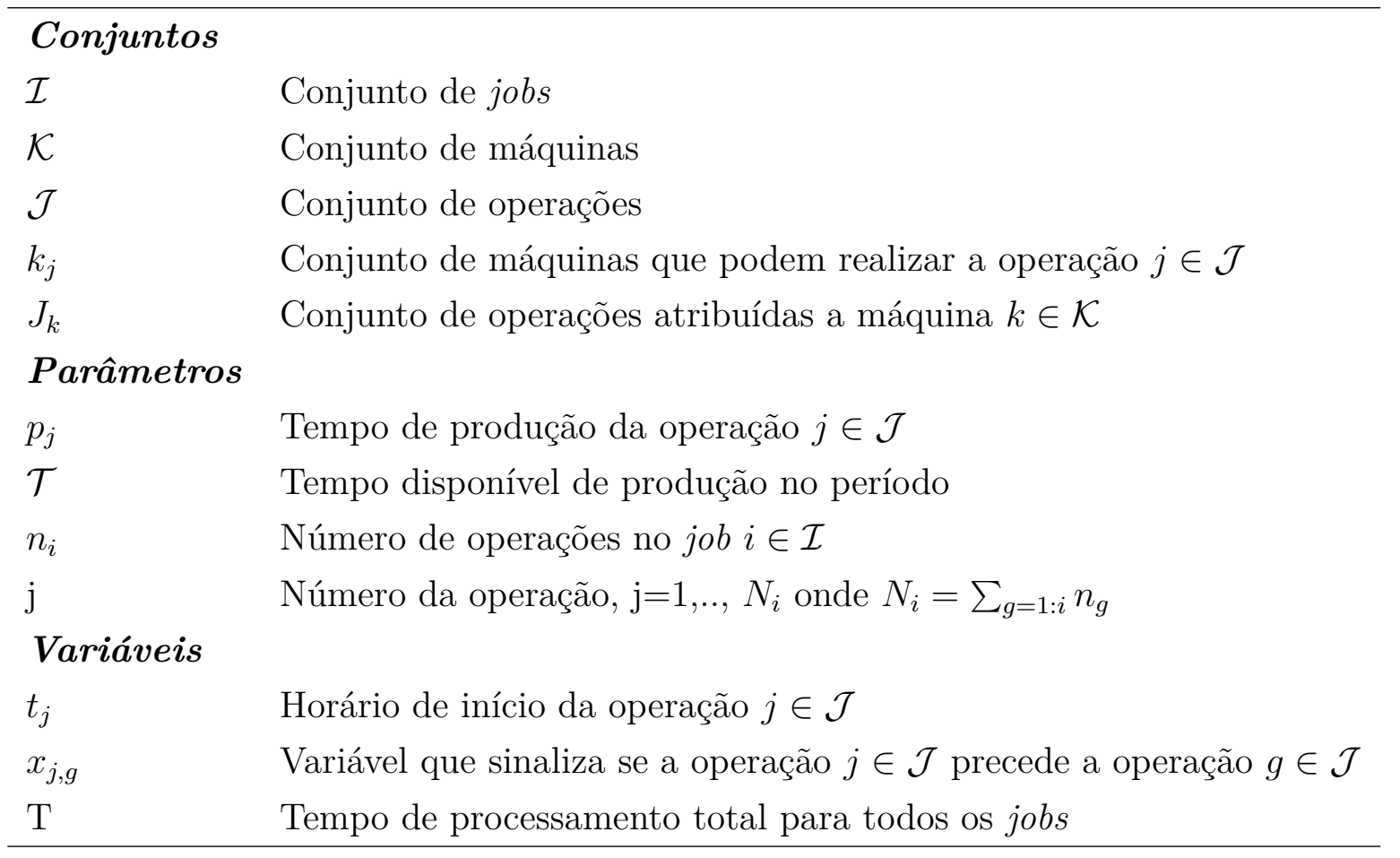

Tabela 4.2: Lista com a nomenclatura contendo os conjuntos, parâmetros e variáveis utilizadas no problema job-shop.

$$
\begin{aligned}
& \underset{t_{j}, x_{j, g}, T}{\operatorname{Minimize}} 0 \\
& \text { subject to: } \\
& \qquad t_{j} \geq t_{j-1}+p_{j-1} \quad \forall i \in I, \forall j \in\left\{N_{i-1}+2, \ldots, N_{i}\right\} \\
& \quad T \geq t_{N_{i}}+p_{N_{i}} \quad \forall i \in I \\
& t_{g} \geq t_{j}+p_{j} x_{j, g}-\mathcal{T}\left(1-x_{j, g}\right) \quad \forall g, j \in J_{k}, \quad \forall k \in \mathcal{K} \\
& t_{j} \geq t_{g}+p_{g}\left(1-x_{j, g}\right)-\mathcal{T} x_{j, g} \quad \forall g, j \in J_{k}, \quad \forall k \in \mathcal{K} \\
& T \leq \mathcal{T} \\
& \quad x_{j, g} \in \begin{cases}0,1\} \\
T, t_{j} \geq 0 \quad \forall j\end{cases}
\end{aligned}
$$

\section{3}

\section{Desenho do experimento}




\subsection{1}

\section{Dados de entrada}

Para avaliar os modelos descritos nas seções (3.1), (3.2), (4.1) e (4.2), um experimento numérico foi desenhado com dados realistas de uma fábrica automotiva. No estudo, foi considerada a produção de 1 produto final $\mathcal{A}$ fabricado a partir de 2 peças $\mathcal{B}$, que por conseguinte são fabricadas a partir duas peças $\mathcal{C}$ cada. Para cada peça $\mathcal{C}$, foi considerada a necessidade de materiais (não fabricados internamente), com consumos de $5,5,1,2$, 1, e 2 itens por produto. Foram consideradas três estações de trabalho $(|\mathcal{M}|=3)$, onde cada uma fabrica um dos produtos $\mathcal{A}, \mathcal{B}$ e $\mathcal{C}$ e o tempo de produção atribuído foi de 1 hora para cada peça. Dividiu-se a produção em 2 faixas $(|\mathcal{K}|=2)$ de capacidades 176 e 88 horas por mês. Essas faixas podem ser interpretadas como capacidade de produção em período de trabalho normal e capacidade de produção em hora-extra.

Não foi considerado um limite na quantidade produzida por dia de forma direta, apenas no tempo de produção. Da mesma forma, a compra de matériaprima não foi limitada. Foram considerados custos de produção $\left(c^{p}\right)$ unitários de $\mathrm{R} \$ 100,00$ e $\mathrm{R} \$ 200,00$ para cada peça em cada faixa $(k \in \mathcal{K})$ respectivamente. O custo de inventário unitário $\left(c^{e}\right)$ atribuído para o produto final $\mathcal{A}$ foi de $\mathrm{R} \$ 50,00$, para cada peça $\mathcal{B} \mathrm{R} \$ 9,00$ e para cada peça $\mathcal{C} \mathrm{R} \$ 4,00$. Para as peças necessárias à fabricação de $\mathcal{C}$ foi considerado um custo unitário de $\mathrm{R} \$ 0,25$. O custo de pedido de matéria-prima, pelo modal "MA" $\left(c^{a}\right)$ e "MB" $\left(c^{b}\right)$, foi de $\mathrm{R} \$ 4,00$ e $\mathrm{R} \$ 2,00$, respectivamente. O custo de falta $\left(c^{d}\right)$ foi considerado como sendo $\mathrm{R} \$ 5000,00$ e o custo de inviabilidade no sequenciamento como $\mathrm{R} \$ 50000,00$ para o produto final $\mathcal{A}$ e $\mathrm{R} \$ 5000,00$ para o produto intermediário $\mathcal{B}$.

Não foi considerado lead time de produção $\left(l^{p}\right)$ fora o tempo de produção de uma hora, foi considerado um lead time de pedido pelo modal "MA" $\left(l^{a}\right)$ de um mês e um lead time de pedido pelo modal "MB" $\left(l^{b}\right)$ de dois meses. Para o inventário inicial, foi considerado peças suficientes para se produzirem 25 produtos finais $\mathcal{A}$. Por considerarmos um produto por máquina, não foi considerado tempo e custo de setup. A previsão da demanda é apresentada na próxima seção.

\subsection{2}

\section{Previsão e Simulação dos Cenários de Demanda}

Tanto a previsão de venda de produto $\mathcal{A}$ para o modelo determinístico, quanto a simulação, para o modelo estocástico, foram obtidas de forma 
semelhante a que está descrita na seção 3.3.2. A única diferença é que a série aqui utilizada foi dividida por 4 em relação a série da seção 3.3.2.

\subsection{3}

\section{Algoritmo de comparação}

As quatro metodologias foram comparadas considerando um planejamento anual com horizonte rolante mensal, congelamento de produção dentro do mês e lead time de dois meses para o pedido por modal "MB" e lead time de um mês para o pedido por modal "MA".

Para realizar a comparação das quatro metodologias foram observados os custos planejados e reais que cada um dos modelos de planejamento/operação incorreriam por mês no decorrer de um ano. O processo elaborado para a comparação está descrito no algoritmo 4 , onde " $N$ " representa o número de simulações que devem ser realizadas.

\section{4}

\section{Resultados Numéricos}

Nesta seção, iremos apresentar os resultados numéricos do experimento desenhado na Seção 4.3 comparando os quatro modelos. Iremos avaliar duas dimensões principais: (i) uma análise out-of-sample, avaliando o impacto financeiro no planejamento da produção seguindo os modelos através da demanda real observada para o ano de 2018 e (ii) uma análise probabilística da performance dos modelos a partir de um novo conjunto de cenários simulados de acordo com a Seção 4.3.2. Considerou-se 30 cenários de demanda para o modelo estocástico. Nas análises que seguem, iremos nos referenciar ao modelo determinístico como M.01, ao modelo estocástico sem sequenciamento como M.02, ao modelo estocástico com sequenciamento incluído ao modelo como M.03 e ao modelo estocástico com verificação de viabilidade do sequenciamento como M.04.

\subsection{1}

\section{Análise Baseada na Demanda Real Observada para 2018}

Utilizando o modelo (M.01), apresentado em (3-1)-(3-9), para o planejamento da produção do ano de 2018 obtivemos um custo total de $\mathrm{R} \$ 778.108,00$. Para o modelo (M.02), apresentado em (3-10)-(3-23), o custo esperado para o ano foi de $\mathrm{R} \$ 720.948,00$, para os modelos estocásticos com sequenciamento interno (M.03) e com heurística para verificação de sequenciamento (M.04) os custos foram $\mathrm{R} \$ 670,792$ e $\mathrm{R} \$ 671,756$, respectivamente. Resultando em uma 


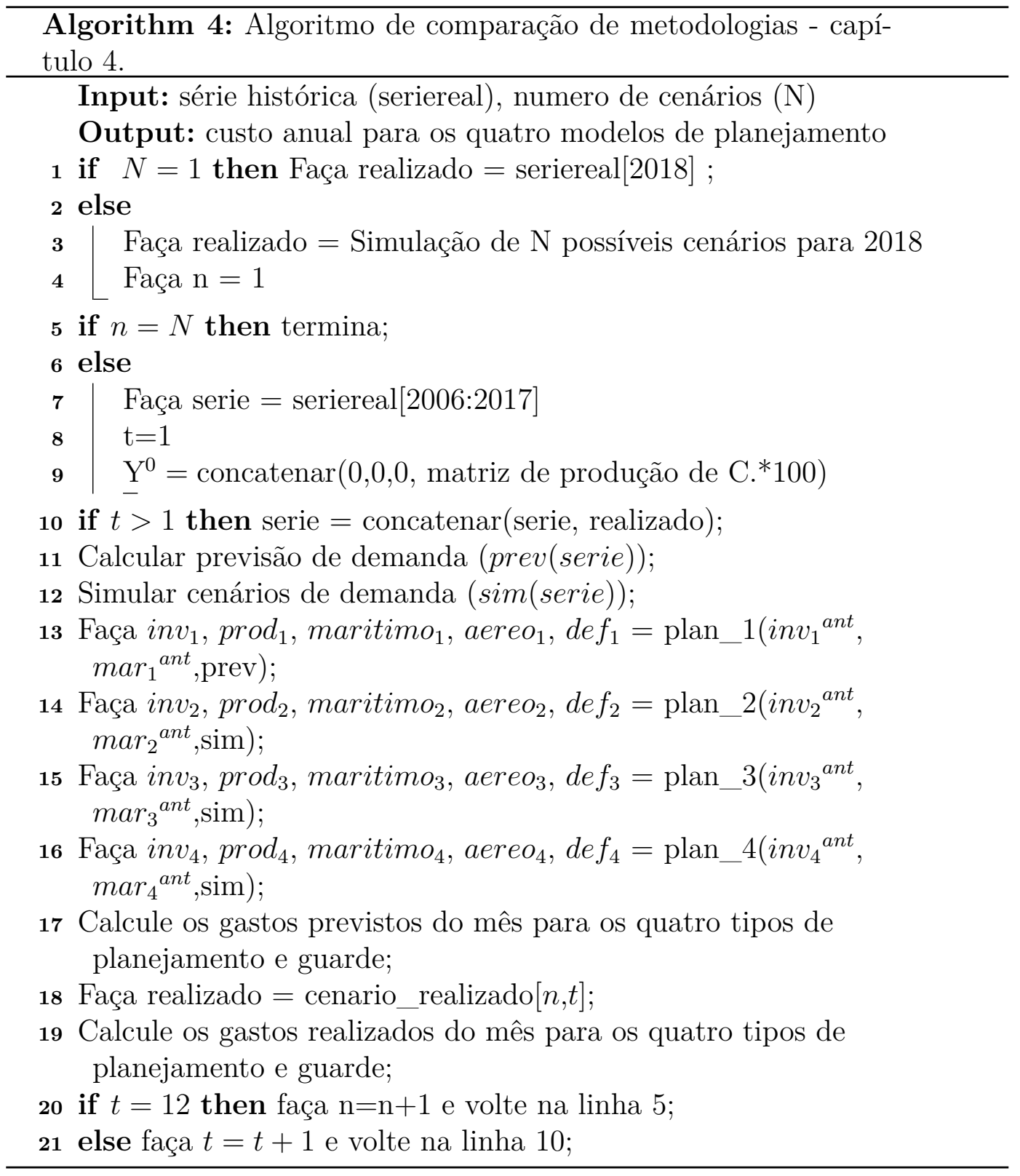

economia (percentual) superior a $13 \%$ entre o primeiro modelo e os dois últimos, e uma economia na ordem de $8 \%$ entre o modelo estocástico sem sequenciamento e o mesmo modelo considerando sequenciamento. Com relação a aderência entre a política planejada e a efetivamente implementada (realizada), no modelo determinístico o custo total realizado foi $38.3 \%$ maior do que o planejado, para o modelo estocástico sem sequenciamento, o acréscimo no custo foi de $15.7 \%$ já para os modelos estocásticos que consideraram o sequenciamento, o acréscimo no custo foi de $6.2 \%$.

A Figura 4.1 apresenta (mês-a-mês) o valor realizado e a diferença entre custo total planejado e o efetivamente observado usando os dados reais para 2018 para cada um dos modelos. 


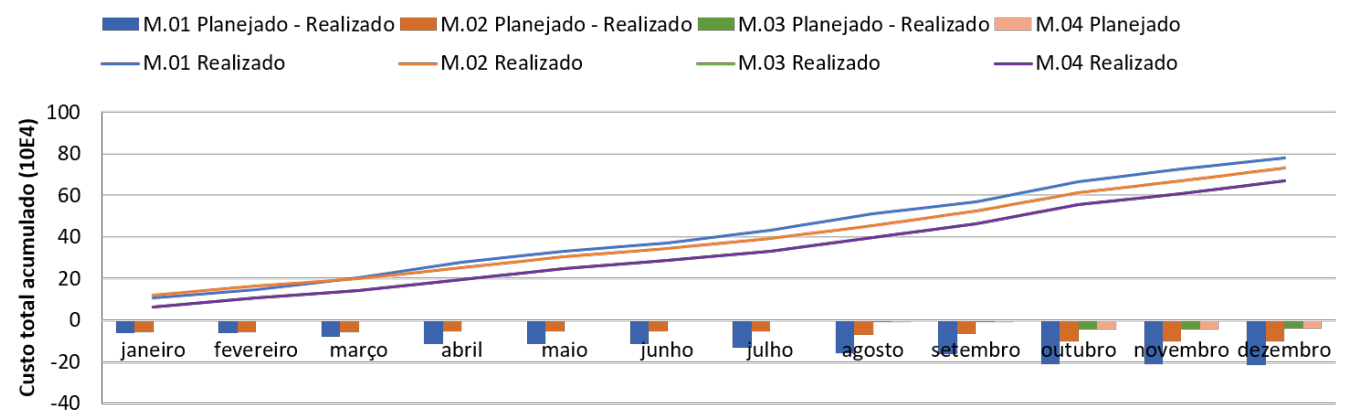

Figura 4.1: Custo total planejado e o efetivamente observado usando os dados reais para 2018 para cada um dos modelos analisados.

\subsection{2}

\section{Análise Probabilística para 2018}

A fim de realizar uma análise probabilística da performance dos modelos com sequenciamento (M.03), (M.04) com relação aos modelos sem sequenciamento, determinístico (M.01) e estocástico (M.02), um novo conjunto de 50 cenários foi simulado. De maneira geral, foi observado que, em média, há uma redução da ordem de 15,6\% no custo total ao se utilizar um dos modelos estocásticos com sequenciamento (M.03) ou (M.04) em relação ao uso do modelo determinístico sem sequenciamento $(M .01)$ e uma redução média na ordem de 9,6\% em relação ao uso do modelo estocástico sem sequenciamento. Na Figura 4.2, a distribuição acumulada (amostral) da diferença percentual entre os custos dos modelos com sequenciamento e sem sequenciamento é apresentada. 


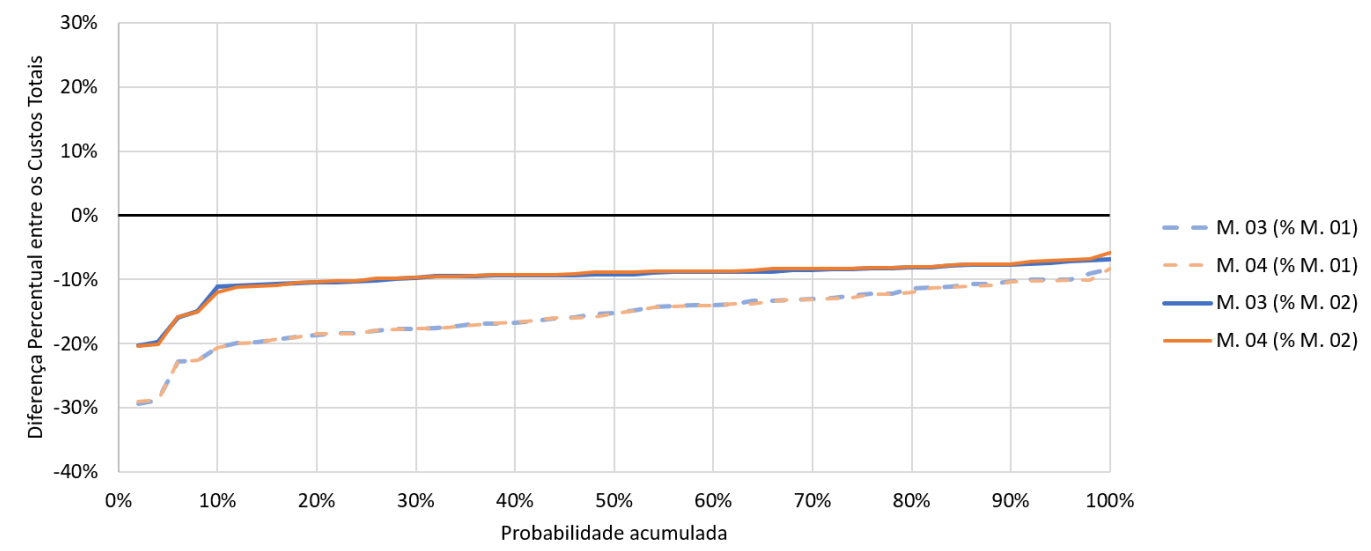

Figura 4.2: Distribuição acumulada (amostral) da diferença percentual entre o custo total dos modelos com sequenciamento M. 03 e M.04 em relação aos modelos sem sequenciamento determinístico M.01 e estocástico M.02

O resultado apresentado no gráfico 4.2 nos indica que em $100 \%$ dos casos houve uma diminuição dos custos com os modelos M.03 e M.04 e estes apresentaram pouca diferença entre si.

Na tabela 4.3 são apresentados os tempos de execução e a memória alocada para rodar uma instância de cada problema.
Modelo Execução Memória
(M.01) $8.725 \mathrm{~ms} \quad$ (15725 allocations: $938.99 \mathrm{KiB})$
(M.02) $132.007 \mathrm{~ms} \quad$ (715568 allocations: $41.20 \mathrm{MiB})$
(M.03) $299.774 \mathrm{~s} \quad(256691434$ allocations: $14.51 \mathrm{GiB})$
(M.04) $102.311 \mathrm{~s} \quad$ (7039710 allocations: $466.60 \mathrm{MiB})$

Tabela 4.3: Tempo de execução e memória utilizada para rodar uma instância de cada modelo. 


\section{5}

\section{Conclusão e Trabalhos Futuros}

No presente trabalho buscou-se avaliar o impacto financeiro e operativo de realizar o planejamento da produção de forma determinística e segregada.

O trabalho foi dividido em dois cases, no primeiro foi proposto um modelo estocástico dois estágios para decisões de compra co-otimizado com o planejamento da produção, no segundo restrições de sequenciamento de jobs também foram consideradas tanto adicionando-as ao modelo anterior, como através de uma heurística para verificação da viabilidade no sequenciamento.

Um modelo com demanda considerada deterministicamente e com pedido de compra feito posteriormente ao planejamento foi utilizado para comparação com os modelos propostos. Foi considerada uma indústria que trabalha com um planejamento anual e horizonte rolante mensal e um estudo numérico com dados realistas do setor automotivo foi realizado para os dois cases.

No primeiro case foram avaliadas três dimensões do problema, na primeira análise foi observada uma economia de aproximadamente $7 \%$ na operação do ano de 2018 com a utilização do modelo proposto. Na segunda análise verificou-se uma redução média de custo na ordem de 3,57\% para 5000 cenários simulados. Na última análise verificou-se que o ganho anual poderia chegar a até $14 \%$, para determinados valores dos parâmetros de custo e tempo avaliados.

No segundo case, para um outro desenho de produção, na operação do ano de 2018 observou-se uma economia superior a 13\% em relação ao modelo determinístico e uma redução no custo na ordem de $8 \%$ em relação ao modelo sem sequenciamento proposto. Numa segunda análise, considerando 50 cenários simulados, verificou-se uma redução média de 15,6\% em relação ao modelo determinístico e uma redução na ordem de $9,6 \%$ em relação ao modelo sem sequenciamento proposto.

Como trabalhos futuros, sugerimos a consideração de produção de produtos variados e uma análise do resultado de um modelo de planejamento multiestágio. 


\section{Referências bibliográficas}

ADAMS, J.; BALAS, E. ; ZAWACK, D.. The shifting bottleneck procedure for job-shop scheduling. Management Science, 34:391-401, 1988.

AXSATER, S.; ROSLING, K.. Multi-level production-inventory control: material requirements planning or reorder point policies. European Journal of Operational Research, 75:405-412, 1994.

P. J. BILLINGTON, J. O. MCCLAIN, L. J. T.. Mathematical programming approaches to capacity-constrained mrp systems: Review, formulation and problem reduction. Management Science, October 1983.

BUSCHKÜHL, L.; SAHLING, F.; HELBER, S. ; TEMPELMEIER, H.. Dynamic capacitated lot-sizing problems: a classification and review of solution approaches. Springer-Verlag, p. 231-261, October 2010.

G. CALLEJA, R. P.. A dispatching algorithm for flexible job-shop scheduling with transfer batches: an industrial application. Production Planning Control, 25:93 - 109, 2014.

CASTELLANO, D.; GEBENNINI, E.; A. GRASSI, T. M. ; RIMINI, B.. Stochastic modeling of a single-vendor single-buyer supply chain with (s, s)inventory policy. IFAC-PapersOnLine, 51:974-979, 2018.

I. A. CHAUDHRY, A. A. K.. A research survey: review of flexible job shop scheduling techniques. International Transactions in Operational Research, 23(3):551-591, 2016.

CHEN, F.; SHENG, Y. S.. Evaluating echelon stock (r, nq) policies in serial production/ inventory systems with stochastic demand. Manag Sci, 40:1262-1275, 1994.

CHEN, F.. Optimal policies for multi-echelon inventory problems with batch ordering. Oper Res, 48:376-389, 2000.

K. Z. GAO, P. N. SUGANTHAN, M. F. T. Q. K. P. Q. Q. S.. Effective ensembles of heuristics for scheduling flexible job shop problem with new job insertion. Computers Industrial Engineering, 90:107 - 117, December 2015. 
Z. HU, G. H.. A two-stage stochastic programming model for lot-sizing and scheduling under uncertainty. International Journal of Production Economics, 180:198-207, 2016.

Z. HU, G. H.. A multi-stage stochastic programming for lot-sizing and scheduling under demand uncertainty. Computers \& Industrial Engineering, 119:157-166, 2018.

R. J. HYNDMAN, G. A.. Forecasting: Principles and Practice. OTexts, Australia, 2nd edition, 2018.

INDERFURTH, K.. How to protect against demandand yield risks in mrp systems. International Journal of Production Research, 121:474-481, February 2007.

JANS, R.; DEGRAEVE, Z.. Modeling industrial lot sizing problems: A review. International Journal of Production Research, 46(6):1619-1643, 2008.

J.JEUNET, N. J.. Measuring the performance of lot-sizing techniques in uncertain environments. International Journal of Production Economics, 64:197-208, March 2000.

KARIMIA, B.; GHOMIA, S. ; WILSONB, J.. The capacitated lot sizing problem: a review of models and algorithms. The International Journal of Management Science, April 2003.

D. KARUNAKARAN, Y. MEI, G. C. M. Z.. Dynamic job shop scheduling under uncertainty using genetic programming. Intelligent and Evolutionary Systems, 8:195 - 210, 2017.

D. H. KROPP, R. C. CARLSON, J. J.. Heuristic lot-sizing approaches for dealing with mrp system nervousness. Decision Sciences, 14:156 - 169, June 2007.

LAGEWEG, B.; LENSTRA, J. ; KAN, A.. Job shop scheduling by implicit enumeration. Management Science, 24:441-450, 1977.

S.N R. LAWRENCE, E. C. S.. Heuristic, optimal, static, and dynamic schedules when processing times are uncertain. Journal of Operations Management, 15(1):71 - 82, February 1997.

LIU, K. C.. Dispatching rules for stochastic finite capacity scheduling. Computers Industrial Engineering, 35:113 - 116, October 1998. 
DÍAZ-MADROÑERO, M.; MULA, J. ; PEIDRO, D.. A review of discrete-time optimization models for tactical production planning. International Journal of Production Research, 52(17):5171-5205,, 2014.

H. MATSUURA, H. TSUBONE, M. K.. Sequencing, dispatching and switching in a dynamic manufacturing environment. International Journal of Production Research, 31(7):1671 - 1688, 1993.

MIN, H.; ZHOU, G.. Supply chain modeling: past, present and future. Computers Industrial Engineering, 43(1-2):231-249, 2002.

J. MULA, R. POLER, J. G. S. F. L.. Models for production planning under uncertainty: A review. International Journal of Production Economics, 103:271-285, February 2006.

MULA, J.; DÍAZ-MADROÑERO, M. ; PEIDRO, D.. A conceptual model for integrating transport planning: Mrp iv. International Federation for Information Processing, 2012.

R. L. NYDICK, H. J. W.. An evaluation of variable-demand lot-sizing techinques. Production and Inventory Management Journal, 30(4):41-48, 1989.

M. PINEDO, G. W.. The "largest variance first" policy in some stochastic scheduling problems. Operations Research, 35(6):799 - 933, December 1987.

PINEDO, M.. Stochastic batch scheduling and the "smallest variance first" rule. Probability in the Engineering and Informational Sciences, 21:579 - 595, October 2007.

POCHET, Y.. "Mathematical Programming Models and Formulations for Deterministic Production Planning Problems." In Computational Combinatorial Optimization, Lecture Notes in Computer Science, edited by M. Jünger and D. Naddef, 57-111. Springer, Berlin, Heidelberg, 2001.

PTAK, C.; SMITH, C.. Orlicky's Material Requirements Planning, Third Edition. McGraw-Hill Education, 2011.

QUADT, D.; KUHN, H.. Capacitated lot-sizing with extensions: a review. Springer-Verlag, p. 61-83, October 2007. 
SALOMON, M.; KROON, L. G.; KUIK, R. ; WASSENHOVE, L. N. V.. Some extensions of the discrete lotsizing and scheduling problem. Management Science, 37(7):751-907, July 1991.

SCHOLLAERT, F.. Money resource planning, MRP-III: the ultimate marriage between business logistics and financial management information systems. Library Albert, 1994.

Y. SHA, J. ZHANG, H. C.. Multistage stochastic programming approach for joint optimization of job scheduling and material ordering under endogenous uncertainties. European Journal of Operational Research, 290(3):886-900, 2021.

SHAPIRO, J. F.. Chapter 8 mathematical programming models and methods for production planning and scheduling, handbooks in operations research and management science. Elsevier, 4:371-443, 1993.

SIMPSON, N. C.. Questioning the relative virtues of dynamic lot sizing rules. Computers Operations Research, 28:899 - 914, August 2001.

C. SUERIE, H. S.. The capacitated lot-sizing problem with linked lot sizes. Management Science, 49(8):983-1119, August 2003.

XU, W.; SONG, D. P.. Integrated optimisation for production capacity, raw material ordering and production planning under time and quantity uncertainties based on two case studies. Operational Research, October 2020.

YUE, D.; YOU, F.. Planning and scheduling of flexible process networks under uncertainty with stochastic inventory: Minlp models and algorithm. AIChE Journal, 59(5):1511-1532, May 2013. 